This item was submitted to Loughborough's Research Repository by the author.

Items in Figshare are protected by copyright, with all rights reserved, unless otherwise indicated.

\title{
Multifractal properties of sample paths of ground state-transformed jump processes
}

PLEASE CITE THE PUBLISHED VERSION

https://doi.org/10.1016/j.chaos.2019.01.008

PUBLISHER

(C) Elsevier

VERSION

AM (Accepted Manuscript)

PUBLISHER STATEMENT

This paper was accepted for publication in the journal Chaos, Solitons and Fractals and the definitive published version is available at https://doi.org/10.1016/j.chaos.2019.01.008.

LICENCE

CC BY-NC-ND 4.0

\section{REPOSITORY RECORD}

Lorinczi, Jozsef, and Xiaochuan Yang. 2019. "Multifractal Properties of Sample Paths of Ground Statetransformed Jump Processes”. figshare. https://hdl.handle.net/2134/36534. 


\title{
MULTIFRACTAL PROPERTIES OF SAMPLE PATHS OF GROUND STATE-TRANSFORMED JUMP PROCESSES
}

\author{
JÓZSEF LŐRINCZI AND XIAOCHUAN YANG
}

\begin{abstract}
We consider a class of Lévy-type processes with unbounded coefficients, arising as Doob $h$-transforms of Feynman-Kac type representations of non-local Schrödinger operators, where the function $h$ is chosen to be the ground state of such an operator. First we show existence of a càdlàg version of the so-obtained ground state-transformed processes. Next we prove that they satisfy a related stochastic differential equation with jumps. Making use of this SDE, we then derive and prove the multifractal spectrum of local Hölder exponents of sample paths of ground state-transformed processes.
\end{abstract}

\section{Introduction}

The purpose of this paper is to investigate the local Hölder continuity properties of sample paths of a class of Lévy-type processes. These processes are obtained through a Doob $h$-transform of random processes occurring in the Feynman-Kac representation of non-local Schrödinger operators. Such processes, and the related operators and non-local equations, are currently much used in a variety of applications, for instance, in models of mathematical physics (anomalous diffusion in porous media, quantum optics etc), however, we will not be concerned with applications in this paper and for a discussion we refer to [23]. Apart from a direct theoretical relevance and applications, sample path regularity properties are also of practical interest in modelling and numerical simulations.

Below we will consider $\mathbb{R}^{d}$-valued Lévy processes $\left(X_{t}\right)_{t \geq 0}$, generated by operators of the form

$$
L f(x)=\sum_{i, j=1}^{d} a_{i j} \frac{\partial^{2} f}{\partial x_{j} \partial x_{i}}(x)+\int_{\mathbb{R}^{d}}\left(f(x+z)-f(x)-1_{\{|z|<1\}}(z) z \cdot \nabla f(x)\right) \nu(z) d z, \quad x \in \mathbb{R}^{d},
$$

with $f \in C_{c}^{\infty}\left(\mathbb{R}^{d}\right)$, and where the matrix $A=\left(a_{i j}\right)_{i, j=1, \ldots, d}$ describes the diffusion part, and the Lévy measure $\nu(\mathrm{d} z)=\nu(z) \mathrm{d} z$ describes the jump part. (For details see Section 2 below.) A landmark example is the fractional Laplacian $L=(-\Delta)^{\alpha / 2}, 0<\alpha<2$, giving rise to an isotropic $\alpha$-stable process, which is a specific case of the class $L=\Psi(-\Delta)$, where $\Psi$ is a Bernstein function. Further cases of much interest include jump-diffusion processes obtained as the sum of a mutually independent Brownian motion and an isotropic $\alpha$-stable process generated by $L=-a \Delta+b(-\Delta)^{\alpha / 2}$, $a, b>0$, isotropic relativistic stable processes generated by $L=\left(-\Delta+m^{2 / \alpha}\right)^{\alpha / 2}-m, m>0$, isotropic geometric $\alpha$-stable processes generated by $L=\log \left(1+(-\Delta)^{\alpha / 2}\right)$, and many others.

Next we consider a suitable class of Borel functions $V: \mathbb{R}^{d} \rightarrow \mathbb{R}$ called potentials, and define the non-local Schrödinger operator $H=-L+V$ and the related semigroup $\left\{e^{-t H}: t \geq 0\right\}$. Assuming

Key-words: jump processes, sample path properties, stochastic differential equations, Hausdorff dimension, Feynman-Kac semigroups, non-local Schrödinger operators, ground states 2010 MS Classification: Primary 60J75, 60G17, 28A78; Secondary 47D08, 47G20 
that $V$ is a Kato-class potential, we then have the Feynman-Kac type representation $[12,32]$

$$
\left(e^{-t H} f\right)(x)=\mathbb{E}^{x}\left[e^{-\int_{0}^{t} V\left(X_{s}\right) d s} f\left(X_{t}\right)\right]=: T_{t} f(x), \quad f \in L^{2}\left(\mathbb{R}^{d}\right), x \in \mathbb{R}^{d}, t \geq 0,
$$

where the expectation is taken with respect to the probability measure of the Lévy process $\left(X_{t}\right)_{t \geq 0}$. The so obtained Feynman-Kac semigroup $\left\{T_{t}: t \geq 0\right\}$ has many convenient properties, allowing a far reaching study of, for instance, spectral properties of $H=-L+V$ or solutions of non-local equations of the type $\partial_{t} u=H u$. However, it is not conservative in the sense that $T_{t} \mathbf{1}_{\mathbb{R}^{d}} \neq \mathbf{1}_{\mathbb{R}^{d}}$, $t>0$, therefore the Lévy process $\left(X_{t}\right)_{t \geq 0}$ perturbed by the function $V$ is in general no longer a random process. Nevertheless, by a suitable Doob $h$-transform one can change the measure under which it becomes a Markov process.

Suppose that $H$ has a non-empty discrete component in its spectrum, and let $\varphi_{0}$ be its unique eigenfunction (called ground state) corresponding to the lowest-lying eigenvalue, i.e., $H \varphi_{0}=\lambda_{0} \varphi_{0}$ with $\varphi_{0} \in \operatorname{Dom} H$ and $\lambda_{0}=\inf \operatorname{Spec} H$. Then the map $f \mapsto \varphi_{0} f$ defines a unitary transform from $L^{2}\left(\mathbb{R}^{d}, \varphi_{0}^{2} d x\right)$ to $L^{2}\left(\mathbb{R}^{d}, d x\right)$. It can be shown, see Section 2 below for further details, that the image $\widetilde{H}$ of $H-\lambda_{0}$ under this unitary map gives the negative of the infinitesimal generator $\widetilde{L}$ of a Markov process, and for suitable test functions we have

$$
\begin{aligned}
(\widetilde{L} f)(x)=\frac{1}{2} \sigma \nabla \cdot \sigma \nabla f(x) & +\sigma \nabla \ln \varphi_{0}(x) \cdot \sigma \nabla f(x)+\int_{0<|z| \leq 1} \frac{\varphi_{0}(x+z)-\varphi_{0}(x)}{\varphi_{0}(x)} z \cdot \nabla f(x) \nu(z) \mathrm{d} z \\
& +\int_{\mathbb{R}^{d} \backslash\{0\}}\left(f(x+z)-f(x)-z \cdot \nabla f(x) \mathbf{1}_{\{|z| \leq 1\}}\right) \frac{\varphi_{0}(x+z)}{\varphi_{0}(x)} \nu(z) \mathrm{d} z, \quad(1.3)
\end{aligned}
$$

where $\nu$ and $A=\sigma \sigma^{T}$ are as in (1.1) above, and where we use the notation $\sigma \nabla \cdot \sigma \nabla f(x)=$ $\sum_{i, j=1}^{d}\left(\sigma \sigma^{T}\right)_{i j} \partial_{x_{i}} \partial_{x_{j}} f(x)$. We call the resulting process a ground state-transformed process (also called $P(\phi)_{1}$-process following the terminology of B. Simon [40]).

The ground state-transformed process is a Lévy-type process resulting from the effect of $V$ giving rise to position-dependent drift and jump components, having almost surely càdlàg paths. However, in contrast with many cases of Lévy-type processes studied in the literature, the coefficients of $\widetilde{L}$ are generally unbounded. It is known that pseudo-differential operators $G$ defined by

$$
(G f)(x)=-\int_{\mathbb{R}^{d}} e^{i x \cdot y} g(x, y) \widehat{f}(y) \mathrm{d} y, \quad f \in C_{\mathrm{c}}^{\infty}\left(\mathbb{R}^{d}\right),
$$

where the hat means Fourier transform, give rise to Lévy-type processes under suitable conditions on the symbol $g(x, y)$. Whenever $C_{\mathrm{c}}^{\infty}\left(\mathbb{R}^{d}\right) \subset \operatorname{Dom} G$ and $G$ generates a Feller process, the Courrège representation

$$
g(x, y)=g(x, 0)-i b(x) \cdot y+\frac{1}{2} y \cdot A(x) y+\int_{\mathbb{R}^{d} \backslash\{0\}}\left(1-e^{i z \cdot y}+i z \cdot y \mathbf{1}_{\{|z| \leq 1\}}\right) \nu(x, \mathrm{~d} z)
$$

holds, where the coefficients $b(x), A(x)$ and $\nu(x, \cdot)$ play the same role of drift vector, diffusion matrix, and jump measure as for Lévy processes, with the essential difference that they are now position dependent $[9,16,8]$. Furthermore, whenever the condition

$$
\sup _{x \in \mathbb{R}^{d}}|g(x, y)| \leq C\left(1+|y|^{2}\right), \quad y \in \mathbb{R}^{d},
$$

holds, with a constant $C>0$, the symbol can be used to analyze various properties of the process generated by $G[37,8]$. It is also known, however, that (1.4) implies that all of the coefficients $b(x), A(x), \nu(x, \cdot)$ are bounded [36]. Recently, there has been an increasing interest in working also with unbounded coefficients, see $[14,30,7,38,31]$ and $[8$, Sect. 3.6]. The results below on ground state-transformed processes complement these efforts since our approach is not through an 
analysis of the symbol, and apart from a direct interest in this context, our class of processes has an immediate relevance in the study of spectral properties of related self-adjoint operators and model Hamiltonians as a bonus [13, 32].

Our concern in the present paper is to study sample path regularity properties of ground statetransformed processes obtained for a large class of operators $H$. The typical long-time behaviour of such processes has been established in [25], which is useful also in characterizing the support of the related Gibbs path measures defined by the right hand side of the Feynman-Kac formula (for perturbations of symmetric $\alpha$-stable processes see also [21]). While the asymptotic behaviour on the long run is driven by the large jumps, regularity at short range depends on the ultraviolet properties of $H$ involving the small jumps. It is reasonable to expect that at least under sufficiently "nice" potentials $V$ the regularity of paths of a ground state-transformed process inherits the regularity of the underlying Lévy process and it does not deteriorate. However, since the drift generated by the perturbation may become rough, the challenge is to establish conditions on $V$ under which path regularity is at least preserved. Results in $[2,43,44]$, where $L=(-\Delta)^{s(x)}$ and $V \equiv 0$, i.e., stable-like processes generated by fractional Laplacians of variable order are considered, indicate that local behaviour may become very complex, and instead of an almost sure rule it can be even dependent on the individual path.

To describe local path regularity, we study the multifractal spectrum of local Hölder exponents of paths. Recall that given a locally bounded function $f: \mathbb{R} \rightarrow \mathbb{R}^{d}$, it is said to belong to the pointwise Hölder space $C^{h}\left(x_{0}\right)$ for $h>0$ and $x_{0} \in \mathbb{R}$ whenever there exist constants $c, \delta>0$, and a polynomial $P$ of $\operatorname{deg} P<\lfloor h\rfloor$ such that for $x \in B\left(x_{0}, \delta\right)$,

$$
\left|f(x)-P\left(x-x_{0}\right)\right| \leq c\left|x-x_{0}\right|^{h} .
$$

The Hölder exponent of $f$ at point $x_{0}$ is then defined by

$$
H_{f}\left(x_{0}\right)=\sup \left\{h>0: f \in C^{h}\left(x_{0}\right)\right\} .
$$

Consider the set

$$
E_{f}(h)=\left\{x \in \mathbb{R}: H_{f}(x)=h\right\} .
$$

The multifractal spectrum of $f$ is the map

$$
D_{f}: h \mapsto \operatorname{dim}_{\mathrm{H}} E_{f}(h),
$$

where $\operatorname{dim}_{H}$ denotes Hausdorff dimension, with the convention that $\operatorname{dim}_{H} \emptyset=-\infty$.

The multifractal spectrum of random processes has been studied by various authors. The above objects are now defined pathwise. For Brownian motion, the Hölder exponent equals $\frac{1}{2}$ everywhere [35], giving

$$
D_{B}(h)= \begin{cases}1 & \text { if } h=\frac{1}{2} \\ -\infty & \text { otherwise }\end{cases}
$$

almost surely, in which case the multifractal reduces to a mono-fractal behaviour. For a general Lévy process $\left(X_{t}\right)_{t \geq 0}$,

$$
X_{t}=b t+\sigma B_{t}+\int_{0}^{t} \int_{|z| \leq 1} z \widetilde{N}(\mathrm{~d} s, \mathrm{~d} z)+\int_{0}^{t} \int_{|z|>1} z N(\mathrm{~d} s, \mathrm{~d} z),
$$

where $b \in \mathbb{R}^{d}$ is the drift term, $\sigma \sigma^{T}$ is the $d \times d$ diffusion matrix, $N$ is a Poisson measure, and $\widetilde{N}$ is the compensated Poisson measure in $\mathbb{R}^{d}$ with intensity given by the Lévy measure $\nu(\mathrm{d} z)$, the 
behaviour relates with the upper Blumenthal-Getoor index [6] given by

$$
\beta_{\nu}=\inf \left\{\gamma \geq 0: \int_{|z| \leq 1}|z|^{\gamma} \nu(\mathrm{d} z)<\infty\right\},
$$

describing the growth rate of the Lévy measure around zero. The integrability condition of Lévy measures implies that $\beta_{\nu} \in[0,2]$. Jaffard [19] has proved the following for a Lévy process with $\beta_{\nu} \in(0,2)$. If $\sigma \neq 0$, then

$$
D_{X}^{1}(h)= \begin{cases}\beta_{\nu} h & \text { if } h<1 / 2 \\ 1 & \text { if } h=1 / 2 \\ -\infty & \text { otherwise }\end{cases}
$$

almost surely, and if $\sigma=0$, then

$$
D_{X}^{2}(h)= \begin{cases}\beta_{\nu} h & \text { if } h \leq 1 / \beta_{\nu} \\ -\infty & \text { otherwise }\end{cases}
$$

almost surely. Balança [1] has shown that the same result holds also for $\beta_{\nu}=2$. (An example of a one-dimensional pure jump Lévy process with $\beta_{\nu}=2$ is one with intensity $\nu(z)=1 /\left(z^{3}|\log z|^{a}\right)$, $a>1$.) Extensions to Lévy fields and time-changed Lévy processes can be found in $[10,3]$.

We also note that there are many further fractal properties of jump processes addressed in the literature. We refer to $[27,34,28,26]$ and the references therein, and for a review see [41].

Our main results are as follows. First, in Theorem 2.1 we prove the existence and basic properties of ground state-transformed processes in the generality considered in this paper. Next in Theorem 3.1, we derive a stochastic differential equation with jumps related to $\widetilde{L}$, and show that the ground state-transformed processes we consider are a weak solution. Using the SDE representation, in Theorem 3.2 we then obtain the multifractal spectrum of local Hölder exponents of our class of processes. We find that whenever the process contains a Brownian component, it has a sweeping effect, and the behaviour is described by (1.7). For cases of pure jump processes, there is a split in the behaviour according to Blumenthal-Getoor indices lower or higher than 1 . For values $\beta_{\nu} \in[1,2]$ the behaviour is described by $(1.8)$, while for $\beta_{\nu} \in(0,1)$ this happens under an increased regularity of the ground state. In Section 3.3 we also discuss the necessity of this extra regularity.

\section{Ground state-transformed jump processes}

\subsection{Lévy processes and perturbations by potentials}

Let $\left(X_{t}\right)_{t>0}$ be a rotationally symmetric Lévy process with values in $\mathbb{R}^{d}, d \geq 1$, i.e., as given by (1.5) in which we set $b=0$. The probability measure of the process starting at $x \in \mathbb{R}^{d}$ will be denoted by $\mathbb{P}^{x}$, and expectation with respect to this measure by $\mathbb{E}^{x}$. The process $\left(X_{t}\right)_{t \geq 0}$ is determined by its characteristic function

$$
\mathbb{E}^{0}\left[e^{i y \cdot X_{t}}\right]=e^{-t \psi(y)}, \quad y \in \mathbb{R}^{d}, t>0,
$$

with the characteristic exponent given by the Lévy-Khintchin formula

$$
\psi(y)=\frac{1}{2} A y \cdot y+\int_{\mathbb{R}^{d}}(1-\cos (y \cdot z)) \nu(\mathrm{d} z) .
$$

Here $A=\left(a_{i j}\right)_{1 \leq i, j \leq d}=\sigma \sigma^{T}$ is a symmetric non-negative definite matrix, and $\nu$ is a symmetric Lévy measure on $\mathbb{R}^{d} \backslash\{0\}$, i.e., $\int_{\mathbb{R}^{d}}\left(1 \wedge|z|^{2}\right) \nu(\mathrm{d} z)<\infty$ and $\nu(E)=\nu(-E)$, for every Borel set $E \subset \mathbb{R}^{d} \backslash\{0\}$, thus the Lévy triplet of the process is $\left(0, \frac{1}{2} A, \nu\right)$. We will assume throughout that the 
Lévy measure in (2.1) has infinite mass and it is absolutely continuous with respect to Lebesgue measure, i.e., $\nu\left(\mathbb{R}^{d} \backslash\{0\}\right)=\infty$ and $\nu(\mathrm{d} x)=\nu(x) \mathrm{d} x$, with density $\nu(x)>0$.

The generator $L$ of the process $\left(X_{t}\right)_{t \geq 0}$ is determined by its symbol $\psi$ through

$$
\widehat{L f}(y)=-\psi(y) \widehat{f}(y), \quad y \in \mathbb{R}^{d}, f \in \operatorname{Dom}(L),
$$

with domain $\operatorname{Dom}(L)=\left\{f \in L^{2}\left(\mathbb{R}^{d}\right): \psi \widehat{f} \in L^{2}\left(\mathbb{R}^{d}\right)\right\}$. It is a negative, non-local, self-adjoint operator with core $C_{\mathrm{c}}^{\infty}\left(\mathbb{R}^{d}\right)$, and it has the expression (1.1) for $f \in C_{\mathrm{c}}^{\infty}\left(\mathbb{R}^{d}\right)$.

Next consider the set of functions

$$
\mathcal{K}^{X}=\left\{f: \mathbb{R} \rightarrow \mathbb{R}^{d}: f \text { is Borel measurable and } \lim _{t \downarrow 0} \sup _{x \in \mathbb{R}^{d}} \mathbb{E}^{x}\left[\int_{0}^{t}\left|f\left(X_{s}\right)\right| d s\right]=0\right\} .
$$

We say that the potential $V: \mathbb{R}^{d} \rightarrow \mathbb{R}$ belongs to $X$-Kato class, i.e., associated with the Lévy process $\left(X_{t}\right)_{t \geq 0}$, whenever it satisfies

$$
V_{-} \in \mathcal{K}^{X} \quad \text { and } \quad V_{+} \in \mathcal{K}_{\text {loc }}^{X}, \quad \text { with } \quad V_{+}=\max \{V, 0\}, V_{-}=\min \{V, 0\},
$$

where $V_{+} \in \mathcal{K}_{\text {loc }}^{X}$ means that $V_{+} 1_{B} \in \mathcal{K}^{X}$, for all compact sets $B \subset \mathbb{R}^{d}$. It is straightforward to see that $L_{\text {loc }}^{\infty}\left(\mathbb{R}^{d}\right) \subset \mathcal{K}_{\text {loc }}^{X}$, moreover, by stochastic continuity of $\left(X_{t}\right)_{t \geq 0}$ also $\mathcal{K}_{\text {loc }}^{X} \subset L_{\text {loc }}^{1}\left(\mathbb{R}^{d}\right)$. Note that $X$-Kato class potentials may have local singularities.

By standard arguments based on Khasminskii's Lemma, see [32, Lem.3.37-3.38], for an $X$-Kato class potential $V$ it follows that there exist suitable constants $C_{1}(X, V), C_{2}(X, V)>0$ such that

$$
\sup _{x \in \mathbb{R}^{d}} \mathbb{E}^{x}\left[e^{-\int_{0}^{t} V\left(X_{s}\right) \mathrm{d} s}\right] \leq \sup _{x \in \mathbb{R}^{d}} \mathbb{E}^{x}\left[e^{\int_{0}^{t} V_{-}\left(X_{s}\right) \mathrm{d} s}\right] \leq C_{1} e^{C_{2} t}, \quad t>0 .
$$

This implies that

$$
T_{t} f(x)=\mathbb{E}^{x}\left[e^{-\int_{0}^{t} V\left(X_{s}\right) \mathrm{d} s} f\left(X_{t}\right)\right], \quad f \in L^{2}\left(\mathbb{R}^{d}\right), t>0,
$$

are well-defined operators. Using the Markov property and stochastic continuity of $\left(X_{t}\right)_{t \geq 0}$, it can be shown that $\left\{T_{t}: t \geq 0\right\}$ is a strongly continuous semigroup of symmetric operators on $L^{2}\left(\mathbb{R}^{d}\right)$, which we call the Feynman-Kac semigroup associated with the process $\left(X_{t}\right)_{t \geq 0}$ and potential $V$. In particular, by the Hille-Yoshida theorem there exists a self-adjoint operator $H$, bounded from below, such that $e^{-t H}=T_{t}$, with core $C_{\mathrm{c}}^{\infty}\left(\mathbb{R}^{d}\right)$. We call the operator $H$ a non-local Schrödinger operator whose kinetic term is the negative of the infinitesimal generator $L$ of the process $\left(X_{t}\right)_{t \geq 0}$. Since any $X$-Kato class potential is relatively form bounded with respect to $-L$ with relative bound less than 1 , we have

$$
H=-L+V,
$$

in form sense, and $V$ acts as a multiplication operator [32, Ch. 3]. For instance, when $\left(X_{t}\right)_{t \geq 0}$ is a subordinate Brownian motion, we have $L=\Psi(-\Delta)$, where $\Psi$ is the Laplace exponent of the corresponding subordinator (given by a Bernstein function), see [12].

We make the following standing assumption throughout this paper.

Assumption 2.1. The self-adjoint operator $H=-L+V$ has a ground state, i.e., there exists an eigenfunction $\varphi_{0} \in \operatorname{Dom} H \subset L^{2}\left(\mathbb{R}^{d}\right)$ such that

$$
H \varphi_{0}=\lambda_{0} \varphi_{0}, \quad \varphi_{0} \not \equiv 0, \quad \lambda_{0}=\inf \operatorname{Spec} H,
$$

and is normalized by $\left\|\varphi_{0}\right\|_{2}=1$.

Remark 2.1. By general results it follows, see [32, Ch.3] and [22, 24], that for the class of operators $L$ and $V$ that we consider, whenever a ground state $\varphi_{0}$ of $H$ does exist, it is unique, has a strictly positive version (which will be chosen throughout below), and it is bounded and continuous, with a 
pointwise decay to zero at infinity. From (2.1)-(2.2) we have that $\operatorname{Spec}(-L)=\operatorname{Spec}_{\mathrm{ess}}(-L)=[0, \infty)$. It also follows by general arguments that whenever the potential is confining, i.e., $V(x) \rightarrow \infty$ as $|x| \rightarrow \infty$, the spectrum of $-L$ completely changes under the perturbation and the spectrum of $H$ becomes purely discrete, consisting of isolated eigenvalues of finite multiplicities. Thus for confining potentials a ground state (2.5) always exists. When the potential is decaying, i.e., $V(x) \rightarrow 0$ as $|x| \rightarrow \infty$, or it is confining in one direction and decaying in another direction, the spectrum of $H$ may or may not contain a discrete component, and the existence of a ground state depends on further details of $V$.

\subsection{Existence and càdlàg property of ground state-transformed processes}

By using $\varphi_{0}>0$, we define the ground state transform as the unitary map

$$
U: L^{2}\left(\mathbb{R}^{d}, \varphi_{0}^{2} \mathrm{~d} x\right) \rightarrow L^{2}\left(\mathbb{R}^{d}, \mathrm{~d} x\right), \quad f \mapsto \varphi_{0} f .
$$

Also, we define the intrinsic Feynman-Kac semigroup

$$
\widetilde{T}_{t} f(x)=\frac{e^{\lambda_{0} t}}{\varphi_{0}(x)} T_{t}\left(\varphi_{0} f\right)(x)
$$

associated with $\left\{T_{t}: t \geq 0\right\}$. Using the integral kernel $u(t, x, y)$ of $T_{t}$, we then have that $\widetilde{T}_{t} f(x)=$ $\int_{\mathbb{R}^{d}} \tilde{u}(t, x, y) f(y) \varphi_{0}^{2}(y) \mathrm{d} y$, with the integral kernel given by

$$
\widetilde{u}(t, x, y)=\frac{e^{\lambda_{0} t} u(t, x, y)}{\varphi_{0}(x) \varphi_{0}(y)}
$$

and infinitesimal generator $\widetilde{L}=-\widetilde{H}$, where

$$
\widetilde{H}=U^{-1}\left(H-\lambda_{0}\right) U
$$

with domain

$$
\text { Dom } \widetilde{H}=\left\{f \in L^{2}\left(\mathbb{R}^{d}, \varphi_{0}^{2} \mathrm{~d} x\right): U f \in \operatorname{Dom} H\right\} .
$$

A calculation gives the expression (1.3), which holds at least for $C_{\mathrm{c}}^{\infty}$ functions. The operators $\widetilde{T}_{t}=e^{t \widetilde{L}}$ are contractions and we have $\widetilde{T}_{t} 1_{\mathbb{R}^{d}}=1_{\mathbb{R}^{d}}$ for all $t \geq 0$, thus $\left\{\widetilde{T}_{t}: t \geq 0\right\}$ is a Markov semigroup on $L^{2}\left(\mathbb{R}^{d}, \varphi_{0}^{2} \mathrm{~d} x\right)$.

The self-adjoint operator $\widetilde{L}$ generates a stationary strong Markov process, which we call ground state-transformed $(G S T)$ process and denote by $\left(\widetilde{X}_{t}\right)_{t \geq 0}$. GST processes have been constructed first for Brownian motion perturbed by potentials, see [40, 5] and [32, Sects. 4.10.2, 4.11.9] for further details and applications. However, due to the jumps in our case there are some essential modifications, and we give a proof of the existence of a càdlàg version of GST jump processes in the generality allowed by Assumption 2.1.

Denote by $\Omega_{\mathrm{r}}$ the space of right continuous functions from $[0, \infty)$ to $\mathbb{R}^{d}$ with left limits (i.e., càdlàg functions), and by $\Omega_{1}$ the space of left continuous functions from $[0, \infty)$ to $\mathbb{R}^{d}$ with right limits (i.e., càglàd functions). Denote the corresponding Borel $\sigma$-fields by $\mathcal{B}\left(\Omega_{\mathrm{r}}\right)$ and $\mathcal{B}\left(\Omega_{1}\right)$, respectively. Also, denote by $\Omega$ the space of càdlàg functions from $\mathbb{R}$ to $\mathbb{R}$, and its Borel $\sigma$-field by $\mathcal{B}(\Omega)$.

Theorem 2.1. Let $\left(X_{t}\right)_{t \geq 0}$ be a Lévy process with generator $L$ as given by (2.1)-(2.2), $V$ be an $X$-Kato class potential, and suppose that $H=-L+V$ has a ground state $\varphi_{0}$. For all $x \in \mathbb{R}^{d}$, there exists a probability measure $\widetilde{\mathbb{P}}^{x}$ on $(\Omega, \mathcal{B}(\Omega))$ and a random process $\left(\widetilde{X}_{t}\right)_{t \in \mathbb{R}}$ satisfying the following properties: 
(1) Let $-\infty<t_{0} \leq t_{1} \leq \ldots \leq t_{n}<\infty$ be an arbitrary division of the real line, for any $n \in \mathbb{N}$. The initial distribution of the process is

$$
\widetilde{\mathbb{P}}^{x}\left(\widetilde{X}_{0}=x\right)=1,
$$

and the finite dimensional distributions of $\widetilde{\mathbb{P}}^{x}$ with respect to the stationary distribution $\varphi_{0}^{2} \mathrm{~d} x$ are given by

$$
\int_{\mathbb{R}^{d}} \mathbb{E}_{\widetilde{\mathbb{P}} x}\left[\prod_{j=0}^{n} f_{j}\left(\widetilde{X}_{t_{j}}\right)\right] \varphi_{0}^{2}(x) \mathrm{d} x=\left(f_{0}, \widetilde{T}_{t_{1}-t_{0}} f_{1} \ldots \widetilde{T}_{t_{n}-t_{n-1}} f_{n}\right)_{L^{2}\left(\mathbb{R}^{d}, \varphi_{0}^{2} \mathrm{~d} x\right)}
$$

for all $f_{0}, f_{n} \in L^{2}\left(\mathbb{R}^{d}, \varphi_{0}^{2} \mathrm{~d} x\right), f_{j} \in L^{\infty}\left(\mathbb{R}^{d}\right), j=1, \ldots, n-1$.

(2) The finite dimensional distributions are time-shift invariant, i.e.,

$$
\int_{\mathbb{R}^{d}} \mathbb{E}_{\widetilde{\mathbb{P}}^{x}}\left[\prod_{j=0}^{n} f_{j}\left(\widetilde{X}_{t_{j}}\right)\right] \varphi_{0}^{2}(x) \mathrm{d} x=\int_{\mathbb{R}^{d}} \mathbb{E}_{\widetilde{\mathbb{P}}^{x}}\left[\prod_{j=0}^{n} f_{j}\left(\widetilde{X}_{t_{j}+s}\right)\right] \varphi_{0}^{2}(x) \mathrm{d} x, \quad s \in \mathbb{R}, n \in \mathbb{N} .
$$

(3) $\left(\widetilde{X}_{t}\right)_{t \geq 0}$ and $\left(\widetilde{X}_{t}\right)_{t \leq 0}$ are independent, and $\widetilde{X}_{-t} \stackrel{\mathrm{d}}{=} \widetilde{X}_{t}$, for all $t \in \mathbb{R}$.

(4) Consider the filtrations $\left(\mathcal{F}_{t}^{+}\right)_{t \geq 0}=\sigma\left(\widetilde{X}_{s}: 0 \leq s \leq t\right)$ and $\left(\mathcal{F}_{t}^{-}\right)_{t \leq 0}=\sigma\left(\widetilde{X}_{s}: t \leq s \leq 0\right)$. Then $\left(\widetilde{X}_{t}\right)_{t \geq 0}$ is a Markov process with respect to $\left(\mathcal{F}_{t}^{+}\right)_{t \geq 0}$, and $\left(\widetilde{X}_{t}\right)_{t \leq 0}$ is a Markov process with respect to $\left(\mathcal{F}_{t}^{-}\right)_{t \leq 0}$.

(5) The map $t \mapsto \widetilde{X}_{t}$ is $\widetilde{\mathbb{P}}^{x}$-almost surely càdlàg.

Furthermore, we have for all $f, g \in L^{2}\left(\mathbb{R}^{d}, \varphi_{0}^{2} \mathrm{~d} x\right)$ the change-of-measure formula

$$
\left(f, \widetilde{T}_{t} g\right)_{L^{2}\left(\mathbb{R}^{d}, \varphi_{0}^{2} \mathrm{~d} x\right)}=\left(f \varphi_{0}, e^{-t\left(H-\lambda_{0}\right)} g \varphi_{0}\right)_{L^{2}\left(\mathbb{R}^{d}, \mathrm{~d} x\right)}=\int_{\mathbb{R}^{d}} \mathbb{E}_{\widetilde{\mathbb{P}}^{x}}\left[f\left(\widetilde{X}_{0}\right) g\left(\widetilde{X}_{t}\right)\right] \varphi_{0}^{2}(x) \mathrm{d} x, \quad t \geq 0 .
$$

The probability measure $\widetilde{\mathbb{P}}^{x}$ is a Gibbs measure on the space of two-sided càdlàg paths, see a discussion for stable processes in [21, Sect. 5.3]. For the remaining part of this section we present a proof of this theorem.

Let $n \in \mathbb{N}$ be arbitrary, and consider any time division $0 \leq t_{0} \leq t_{1} \leq \ldots \leq t_{n}$. Define the set function $P_{\left\{t_{0}, \ldots, t_{n}\right\}}: \times_{j=0}^{n} \mathcal{B}\left(\mathbb{R}^{d}\right) \rightarrow \mathbb{R}$ by

$$
\begin{aligned}
& P_{\left\{t_{0}\right\}}\left(A_{0}\right)=\left(\mathbf{1}, \widetilde{T}_{t_{0}} \mathbf{1}_{A_{0}}\right)_{L^{2}\left(\mathbb{R}^{d}, \varphi_{0}^{2} \mathrm{~d} x\right)}=\left(\mathbf{1}, \mathbf{1}_{A_{0}}\right)_{L^{2}\left(\mathbb{R}^{d}, \varphi_{0}^{2} \mathrm{~d} x\right)} \\
& P_{\left\{t_{0}, \ldots, t_{n}\right\}}\left(\times_{j=0}^{n} A_{j}\right)=\left(\mathbf{1}_{A_{0}}, \widetilde{T}_{t_{1}-t_{0}} \mathbf{1}_{A_{1}} \ldots \widetilde{T}_{t_{n}-t_{n-1}} \mathbf{1}_{A_{n}}\right)_{L^{2}\left(\mathbb{R}^{d}, \varphi_{0}^{2} \mathrm{~d} x\right)}, \quad n \in \mathbb{N},
\end{aligned}
$$

with arbitrary Borel sets $A_{0}, \ldots, A_{n} \in \mathcal{B}\left(\mathbb{R}^{d}\right)$.

Step 1: First we obtain a probability measure on the set of all functions $[0, \infty) \rightarrow \mathbb{R}^{d}$ by a projective limit of the prescribed finite dimensional distributions (2.11)-(2.12), which is a standard step. Denote the set of finite subsets of the positive semi-axis by $\mathcal{P}_{f}\left(\mathbb{R}^{+}\right)=\{\Lambda \subset[0, \infty):|\Lambda|<\infty\}$, where the bars denote cardinality of the set. It can be verified directly that the family of set functions $\left(P_{\Lambda}\right)_{\Lambda \in \mathcal{P}_{f}\left(\mathbb{R}^{+}\right)}$satisfies the consistency condition of the marginals

$$
P_{\left\{t_{0}, \ldots, t_{n+m}\right\}}\left(\left(\times_{j=0}^{n} A_{j}\right) \times\left(\times_{j=n+1}^{n+m} \mathbb{R}^{d}\right)\right)=P_{\left\{t_{0}, \ldots, t_{n}\right\}}\left(\times_{j=0}^{n} A_{j}\right), \quad n, m \in \mathbb{N} .
$$

Hence by the Kolmogorov extension theorem there exists a probability measure $P_{\infty}$ and a random process $\left(Z_{t}\right)_{t \geq 0}$ on the measurable space $\left(\left(\mathbb{R}^{d}\right)^{[0, \infty)}, \sigma(\mathcal{A})\right)$, where

$$
\mathcal{A}=\left\{\omega: \mathbb{R} \rightarrow \mathbb{R}^{d}:\left.\operatorname{Ran} \omega\right|_{\Lambda} \subset E, E \in\left(\mathcal{B}\left(\mathbb{R}^{d}\right)\right)^{|\Lambda|}, \Lambda \in \mathcal{P}_{f}\left(\mathbb{R}^{+}\right)\right\},
$$


such that

$$
P_{\left\{t_{0}\right\}}(A)=\mathbb{E}_{P_{\infty}}\left[\mathbf{1}_{A}\left(Z_{t_{0}}\right)\right] \quad \text { and } \quad P_{\left\{t_{0}, \ldots, t_{n}\right\}}\left(\times_{j=0}^{n} A_{j}\right)=\mathbb{E}_{P_{\infty}}\left[\prod_{j=0}^{n} \mathbf{1}_{A_{j}}\left(Z_{t_{j}}\right)\right], \quad n \in \mathbb{N},
$$

hold. Hence we have

$$
\begin{aligned}
& \mathbb{E}_{P_{\infty}}\left[f_{0}\left(Z_{t_{0}}\right)\right]=\left(1, \widetilde{T}_{t_{0}} f_{0}\right)_{L^{2}\left(\mathbb{R}^{d}, \varphi_{0}^{2} \mathrm{~d} x\right)}=\left(\mathbf{1}, f_{0}\right)_{L^{2}\left(\mathbb{R}^{d}, \varphi_{0}^{2} \mathrm{~d} x\right)} \\
& \mathbb{E}_{P_{\infty}}\left[\prod_{j=0}^{n} f_{j}\left(Z_{t_{j}}\right)\right]=\left(f_{0}, \widetilde{T}_{t_{1}-t_{0}} f_{1} \ldots \widetilde{T}_{t_{n}-t_{n-1}} f_{n}\right)_{L^{2}\left(\mathbb{R}^{d}, \varphi_{0}^{2} \mathrm{~d} x\right)},
\end{aligned}
$$

for $f_{j} \in L^{\infty}\left(\mathbb{R}^{d}\right), j=1, \ldots, n-1, f_{0}, f_{n} \in L^{2}\left(\mathbb{R}^{d}, \varphi_{0}^{2} \mathrm{~d} x\right), 0 \leq t_{0} \leq t_{1} \leq \ldots \leq t_{n}$, and all $n \in \mathbb{N}$.

Step 2: Next we prove the existence of both a càdlàg and a càglàd version of $\left(Z_{t}\right)_{t \geq 0}$. In this step we show that the Dynkin-Kinney condition holds.

Lemma 2.1. Let $T>0$ be arbitrary but fixed. Then for every $\varepsilon>0$ we have $P_{\infty}\left(\left|Z_{t}-Z_{s}\right|>\varepsilon\right) \rightarrow 0$ as $|t-s| \rightarrow 0$, uniformly in $s, t \in[0, T]$.

Proof. We write the right hand side of (2.15) in terms of $\left(X_{t}\right)_{t \geq 0}$, i.e.,

$$
\mathbb{E}_{P_{\infty}}\left[\prod_{j=0}^{n} f_{j}\left(Z_{t_{j}}\right)\right]=\int_{\mathbb{R}^{d}} \mathbb{E}^{x}\left[e^{-\int_{0}^{t_{n}}\left(V\left(X_{s}\right)-\lambda_{0}\right) \mathrm{d} s}\left(\prod_{j=0}^{n} f_{j}\left(X_{t_{j}}\right)\right) \varphi_{0}\left(X_{t_{n}}\right)\right] \varphi_{0}(x) \mathrm{d} x .
$$

Let $0 \leq s<t \leq T$, and denote by $B_{\varepsilon}(x)$ the ball of radius $\varepsilon$ centered in $x$. Notice that by $(2.15)$ we have

$$
P_{\infty}\left(\left|Z_{t}-Z_{s}\right|>\varepsilon\right)=\left(\mathbf{1}, \widetilde{T}_{t-s} \mathbf{1}_{B_{\varepsilon}(0)^{c}}\right)_{L^{2}\left(\mathbb{R}^{d}, \varphi_{0}^{2} \mathrm{~d} x\right)}
$$

Recall that $\mathbb{E}^{x}$ is expectation with respect to the measure $\mathbb{P}^{x}$ of the Lévy process $\left(X_{t}\right)_{t \geq 0}$. By (2.16), the Markov property of $\left(X_{t}\right)_{t \geq 0}$, and the conservative property of the intrinsic semigroup $\left\{\widetilde{T}_{t}: t \geq 0\right\}$ on $L^{2}\left(\mathbb{R}^{d}, \varphi_{0}^{2} \mathrm{~d} x\right)$, we furthermore have

$$
P_{\infty}\left(\left|Z_{t}-Z_{s}\right|>\varepsilon\right)=\int_{\mathbb{R}^{d}} \mathbb{E}^{x}\left[e^{-\int_{0}^{t-s}\left(V\left(X_{r}\right)-\lambda_{0}\right) \mathrm{d} r} \varphi_{0}\left(X_{t-s}\right) \mathbf{1}_{B_{\varepsilon}(x)^{c}}\left(X_{t-s}\right)\right] \varphi_{0}(x) \mathrm{d} x .
$$

Schwarz inequality gives

$$
\begin{aligned}
& P_{\infty}\left(\left|Z_{t}-Z_{s}\right|>\varepsilon\right) \\
& \leq \int_{\mathbb{R}^{d}}\left(\mathbb{E}^{x}\left[\varphi_{0}^{2}\left(X_{t-s}\right)\right]\right)^{1 / 2}\left(\mathbb{E}^{x}\left[\mathbf{1}_{B_{\varepsilon}(x)^{c}}\left(X_{t-s}\right) e^{-2 \int_{0}^{t-s}\left(V\left(X_{r}\right)-\lambda_{0}\right) \mathrm{d} r}\right]\right)^{1 / 2} \varphi_{0}(x) \mathrm{d} x .
\end{aligned}
$$

Using again Schwarz inequality, we have

$$
\begin{aligned}
\mathbb{E}^{x} & {\left[\mathbf{1}_{B_{\varepsilon}(x)^{c}}\left(X_{t-s}\right) e^{-2 \int_{0}^{t-s}\left(V\left(X_{r}\right)-\lambda_{0}\right) \mathrm{d} r}\right] } \\
& \leq\left(\mathbb{E}^{x}\left[\mathbf{1}_{B_{\varepsilon}(x)^{c}}\left(X_{t-s}\right)\right]\right)^{1 / 2}\left(\mathbb{E}^{x}\left[e^{-4 \int_{0}^{t-s}\left(V\left(X_{r}\right)-\lambda_{0}\right) \mathrm{d} r}\right]\right)^{1 / 2} \leq C \mathbb{P}\left(\left|X_{t-s}\right|>\varepsilon\right)^{1 / 2},
\end{aligned}
$$

where $C=\sup _{x \in \mathbb{R}^{d}}\left(\mathbb{E}^{x}\left[e^{-4 \int_{0}^{t-s}\left(V\left(X_{r}\right)-\lambda_{0}\right) \mathrm{d} r}\right]\right)^{1 / 2}$. Thus by (2.17) and a repeated use of Schwarz inequality, we get

$$
P_{\infty}\left(\left|Z_{t}-Z_{s}\right|>\varepsilon\right) \leq C^{1 / 2} \mathbb{P}\left(\left|X_{t-s}\right|>\varepsilon\right)^{1 / 4}\left\|\varphi_{0}\right\|_{2}^{2},
$$

which goes to zero as $|t-s| \rightarrow 0$ by stochastic continuity of $\left(X_{t}\right)_{t \geq 0}$.

To show uniform convergence, fix $\eta>0$. By stochastic continuity, for every $t$ there exists $\rho_{t}>0$ such that $P_{\infty}\left(\left|Z_{t}-Z_{s}\right| \geq \frac{\varepsilon}{2}\right) \leq \frac{\eta}{2}$ for $|s-t|<\rho_{t}$. Let $I_{t}=\left(t-\frac{\rho_{t}}{2}, t+\frac{\rho_{t}}{2}\right)$. There is a finite covering 
$I_{t_{j}}, j=1, \ldots, n$, such that $\cup_{j=1}^{n} I_{t_{j}} \supset[0, T]$. Let $\rho=\min _{1 \leq j \leq n} \rho_{t_{j}}$. If $|s-t|<\rho$ and $s, t \in[0, T]$, then $t \in I_{t_{j}}$ for some $j$, hence $\left|s-t_{j}\right|<\rho_{t_{j}}$ and

$$
P_{\infty}\left(\left|Z_{t}-Z_{s}\right|>\varepsilon\right) \leq P_{\infty}\left(\left|Z_{t}-Z_{t_{j}}\right|>\varepsilon / 2\right)+P_{\infty}\left(\left|Z_{s}-Z_{t_{j}}\right|>\varepsilon / 2\right)<\eta .
$$

Recall that $\Omega_{\mathrm{r}}$ and $\Omega_{1}$ denote the càdlàg and càglàd path spaces over $[0, \infty)$, respectively. By Lemma 2.1, there exists a càdlàg version $\bar{Z}=\left(\bar{Z}_{t}\right)_{t \geq 0}$ of $\left(Z_{t}\right)_{t \geq 0}$ on the space $\left(\left(\mathbb{R}^{d}\right)^{[0, \infty)}, \sigma(\mathcal{A}), P_{\infty}\right)$. Denote the image measure of $P_{\infty}$ on $\left(\Omega_{\mathrm{r}}, \mathcal{B}\left(\Omega_{\mathrm{r}}\right)\right)$ by $Q_{\mathrm{r}}=P_{\infty} \circ \bar{Z}^{-1}$. Let $\left(Y_{t}\right)_{t \geq 0}$ be the coordinate process on $\left(\Omega_{\mathrm{r}}, \mathcal{B}\left(\Omega_{\mathrm{r}}\right), Q_{\mathrm{r}}\right)$ such that $\bar{Z}_{t} \stackrel{\mathrm{d}}{=} Y_{t}$. In terms of $\left(Y_{t}\right)_{t \geq 0}$, equalities $(2.14)-(2.15)$ become

$$
\begin{aligned}
& \left(\mathbf{1}, \widetilde{T}_{t_{0}} f_{0}\right)_{L^{2}\left(\mathbb{R}^{d}, \varphi_{0}^{2} \mathrm{~d} x\right)}=\left(\mathbf{1}, f_{0}\right)_{L^{2}\left(\mathbb{R}^{d}, \varphi_{0}^{2} \mathrm{~d} x\right)}=\mathbb{E}_{Q_{\mathrm{r}}}\left[f_{0}\left(Y_{t_{0}}\right)\right], \\
& \left(f_{0}, \widetilde{T}_{t_{1}-t_{0}} f_{1} \ldots f_{n-1} \widetilde{T}_{t_{n}-t_{n-1}} f_{n}\right)_{L^{2}\left(\mathbb{R}^{d}, \varphi_{0}^{2} \mathrm{~d} x\right)}=\mathbb{E}_{Q_{\mathrm{r}}}\left[\prod_{j=0}^{n} f_{j}\left(Y_{t_{n}}\right)\right] .
\end{aligned}
$$

Similarly, a càglàd version $\underline{Z}=\left(\underline{Z}_{t}\right)_{t \geq 0}$ of $\left(Z_{t}\right)_{t \geq 0}$ can be also constructed on the same probability space $\left(\left(\mathbb{R}^{d}\right)^{[0, \infty)}, \sigma(\mathcal{A}), P_{\infty}\right)$. Likewise, there exists a probability measure $Q_{1}$ on the càglàd space $\left(\Omega_{1}, \mathcal{B}\left(\Omega_{1}\right)\right.$ such that the coordinate process $\left(Y_{t}\right)_{t \geq 0}$ satisfies $(2.18)-(2.19)$ with $Q_{1}$, and $Q_{1}=P_{\infty} \circ \underline{Z}^{-1}$ holds.

Step 3: We define the regular conditional probability measures $Q_{\mathrm{r}}^{x}(\cdot)=Q_{\mathrm{r}}\left(\cdot \mid Y_{0}=x\right)$ and $Q_{1}^{x}(\cdot)=$ $Q_{1}\left(\cdot \mid Y_{0}=x\right)$ for $x \in \mathbb{R}^{d}$ on $\left(\Omega_{\mathrm{r}}, \mathcal{B}\left(\Omega_{\mathrm{r}}\right)\right)$ and $\left(\Omega_{1}, \mathcal{B}\left(\Omega_{1}\right)\right)$, respectively. Since $Y_{0}$ is distributed by $\varphi_{0}^{2}(x) \mathrm{d} x$, we have $Q_{\mathrm{r}}(A)=\int_{\mathbb{R}^{d}} \mathbb{E}_{Q_{\mathrm{r}}^{x}}\left[\mathbf{1}_{A}\right] \varphi_{0}^{2}(x) \mathrm{d} x$ and $Q_{\mathrm{l}}(A)=\int_{\mathbb{R}^{d}} \mathbb{E}_{Q_{1}^{x}}\left[\mathbf{1}_{A}\right] \varphi_{0}^{2}(x) \mathrm{d} x$. Hence the process $\left(Y_{t}\right)_{t \geq 0}$ on $\left(\Omega_{\mathrm{r}}, \mathcal{B}\left(\Omega_{\mathrm{r}}\right), Q_{\mathrm{r}}^{x}\right)$ satisfies

$$
\begin{aligned}
& \left(\mathbf{1}, \widetilde{T}_{t_{0}} f_{0}\right)_{L^{2}\left(\mathbb{R}^{d}, \varphi_{0}^{2} \mathrm{~d} x\right)}=\left(\mathbf{1}, f_{0}\right)_{L^{2}\left(\mathbb{R}^{d}, \varphi_{0}^{2} \mathrm{~d} x\right)}=\int_{\mathbb{R}^{d}} \mathbb{E}_{Q_{\mathrm{r}}^{x}}\left[f_{0}\left(Y_{t_{0}}\right)\right] \varphi_{0}^{2}(x) \mathrm{d} x \\
& \left(f_{0}, \widetilde{T}_{t_{1}-t_{0}} f_{1} \ldots f_{n-1} \widetilde{T}_{t_{n}-t_{n-1}} f_{n}\right)_{L^{2}\left(\mathbb{R}^{d}, \varphi_{0}^{2} \mathrm{~d} x\right)}=\int_{\mathbb{R}^{d}} \mathbb{E}_{Q_{\mathrm{r}}^{x}}\left[\prod_{j=0}^{n} f_{j}\left(Y_{t_{j}}\right)\right] \varphi_{0}^{2}(x) \mathrm{d} x,
\end{aligned}
$$

and the process $\left(Y_{t}\right)_{t \geq 0}$ on $\left(\Omega_{1}, \mathcal{B}\left(\Omega_{1}\right), Q_{1}^{x}\right)$ satisfies $(2.20)-(2.21)$ with $Q_{1}^{x}$.

Lemma 2.2. The coordinate process $\left(Y_{t}\right)_{t \geq 0}$ is a Markov process on $\left(\Omega_{\mathrm{r}}, \mathcal{B}\left(\Omega_{\mathrm{r}}\right), Q_{\mathrm{r}}^{x}\right)$ with respect to the natural filtration $\left(\mathcal{F}_{t}\right)_{t \geq 0}$. Similarly, the coordinate process $\left(Y_{t}\right)_{t \geq 0}$ is a Markov process on the probability space $\left(\Omega_{1}, \mathcal{B}\left(\Omega_{1}\right), Q_{1}^{x}\right)$ with respect to the natural filtration $\left(\mathcal{F}_{t}\right)_{t \geq 0}$.

Proof. Let $q_{t}(x, A)=\widetilde{T}_{t} \mathbf{1}_{A}(x)$, for every $A \in \mathcal{B}\left(\mathbb{R}^{d}\right), x \in \mathbb{R}^{d}$ and $t \geq 0$. Clearly, $q_{t}(x, A)=$ $\mathbb{E}_{Q_{\mathrm{r}}^{x}}\left[\mathbf{1}_{A}\left(Y_{t}\right)\right]$, and by (2.20)-(2.21) the finite dimensional distributions of $\left(Y_{t}\right)_{t \geq 0}$ can be written as

$$
\mathbb{E}_{Q_{\mathrm{r}}^{x}}\left[\prod_{j=0}^{n} \mathbf{1}_{A_{j}}\left(Y_{t_{j}}\right)\right]=\int_{\mathbb{R}^{d}} \prod_{j=0}^{n} \mathbf{1}_{A_{j}}\left(x_{j}\right) q_{t_{j}-t_{j-1}}\left(x_{j-1}, \mathrm{~d} x_{j}\right),
$$

with $t_{0}=0$ and $x_{0}=x$. By using the properties of the semigroup $\left\{\widetilde{T}_{t}: t \geq 0\right\}$, it is checked directly that $q_{t}(x, A)$ is a probability transition kernel, thus $\left(Y_{t}\right)_{t \geq 0}$ is a Markov process with finite dimensional distributions given by (2.22). The second statement can be proven similarly.

Step 4: Next we construct a random process indexed by the whole real line $\mathbb{R}$. Consider $\widehat{\Omega}=\Omega_{\mathrm{r}} \times \Omega_{1}$, with product $\sigma$-field $\widehat{\mathcal{F}}=\mathcal{B}\left(\Omega_{\mathrm{r}}\right) \times \mathcal{B}\left(\Omega_{1}\right)$ and product measure $\widehat{Q}^{x}=Q_{\mathrm{r}}^{x} \times Q_{1}^{x}$. Define the coordinate process $\left(\widehat{Y}_{t}\right)_{t \geq 0}$ by

$$
\widehat{Y}_{t}(\omega)= \begin{cases}\omega_{1}(t) & t \geq 0 \\ \omega_{2}(-t) & t<0\end{cases}
$$


for $\omega=\left(\omega_{1}, \omega_{2}\right) \in \widehat{\Omega}$. This is then a random process $\left(\widehat{Y}_{t}\right)_{t \in \mathbb{R}}$ on $\left(\widehat{\Omega}, \widehat{\mathcal{F}}, \widehat{Q}^{x}\right)$ such that $\widehat{Q}^{x}\left(\widehat{Y}_{0}=x\right)=1$, and $\mathbb{R} \ni t \mapsto \widehat{Y}_{t}(\omega)$ is càdlàg. It is direct to see that $\widehat{Y}_{t}, t \geq 0$, and $\widehat{Y}_{s}, s \leq 0$, are independent, and $\widehat{Y}_{t} \stackrel{\mathrm{d}}{=} \widehat{Y}_{-t}$.

Step 5: Denote the image measure of $\widehat{Q}^{x}$ on $\left(\Omega_{\mathrm{r}}, \mathcal{B}\left(\Omega_{\mathrm{r}}\right)\right)$ with respect to $\widehat{Y}=\left(\widehat{Y}_{t}\right)_{t \in \mathbb{R}}$ by $\widetilde{\mathbb{P}}^{x}=$ $\widehat{Q}^{x} \circ \widehat{Y}^{-1}$. Let $\widetilde{X}_{t}(\omega)=\omega(t), t \in \mathbb{R}, \omega \in \Omega$, denote the coordinate process. Clearly, we have $\widetilde{X}_{t} \stackrel{\mathrm{d}}{=} Y_{t}$ for $t \in \mathbb{R}$. Thus we see that $\widetilde{X}_{t} \stackrel{\mathrm{d}}{=} \widetilde{X}_{-t}$, and by Step 4 above $\left(\widetilde{X}_{t}\right)_{t \geq 0}$ and $\left(\widetilde{X}_{t}\right)_{t \leq 0}$ are independent. Furthermore, by Step 2 we have that $\left(\widetilde{X}_{t}\right)_{t \geq 0}$ and $\left(\widetilde{X}_{t}\right)_{t \leq 0}$ are Markov processes with respect to $\left(\mathcal{F}_{t}^{+}\right)_{t \geq 0}$ and $\left(\mathcal{F}_{t}^{-}\right)_{t \leq 0}$, respectively.

To prove shift invariance, consider arbitrary time-points $t_{0} \leq \ldots \leq t_{n} \leq 0 \leq t_{n+1} \leq \ldots \leq t_{n+m}$, $n, m \in \mathbb{N}$. Then by independence of $\left(\widetilde{X}_{t}\right)_{t \leq 0}$ and $\left(\widetilde{X}_{t}\right)_{t \geq 0}$ we have

$$
\int_{\mathbb{R}^{d}} \mathbb{E}_{\widetilde{\mathbb{P}}^{x}}\left[\prod_{j=0}^{n+m} f_{j}\left(\widetilde{X}_{t_{j}}\right)\right] \varphi_{0}^{2} \mathrm{~d} x=\int_{\mathbb{R}^{d}} \mathbb{E}_{\widetilde{\mathbb{P}}^{x}}\left[\prod_{j=0}^{n} f_{j}\left(\widetilde{X}_{t_{j}}\right)\right] \mathbb{E}_{\widetilde{\mathbb{P}}^{x}}\left[\prod_{j=n+1}^{n+m} f_{j}\left(\widetilde{X}_{t_{j}}\right)\right] \varphi_{0}^{2} \mathrm{~d} x
$$

Moreover,

$$
\mathbb{E}_{\widetilde{\mathbb{P}} x}\left[\prod_{j=n+1}^{n+m} f_{j}\left(\widetilde{X}_{t_{j}}\right)\right]=\left(\widetilde{T}_{t_{n+1}} f_{n+1} \widetilde{T}_{t_{n+2}-t_{n+1}} f_{n+2} \ldots \widetilde{T}_{t_{n+m}-t_{n+m-1}} f_{n+m}\right)(x)
$$

and

$$
\mathbb{E}_{\widetilde{\mathbb{P}} x}\left[\prod_{j=0}^{n} f_{j}\left(\widetilde{X}_{t_{j}}\right)\right]=\mathbb{E}_{\widetilde{\mathbb{P}} x}\left[\prod_{j=0}^{n} f_{j}\left(\widetilde{X}_{-t_{j}}\right)\right]=\left(\widetilde{T}_{-t_{n}} f_{n} \widetilde{T}_{t_{n}-t_{n-1}} f_{n-1} \ldots \widetilde{T}_{t_{1}-t_{0}} f_{0}\right)(x) .
$$

A combination of the above gives

$$
\begin{aligned}
\int_{\mathbb{R}^{d}} \mathbb{E}_{\widetilde{\mathbb{P}} x}\left[\prod_{j=0}^{n+m} f_{j}\left(\widetilde{X}_{t_{j}}\right)\right] \varphi_{0}^{2}(x) \mathrm{d} x & =\left(\widetilde{T}_{-t_{n}} f_{n} \ldots \widetilde{T}_{t_{1}-t_{0}} f_{0}, \widetilde{T}_{t_{n+1}} f_{n+1} \ldots \widetilde{T}_{t_{n+m}-t_{n+m-1}} f_{n+m}\right)_{L^{2}\left(\mathbb{R}^{d}, \varphi_{0}^{2} \mathrm{~d} x\right)} \\
& =\left(f_{0}, \widetilde{T}_{t_{1}-t_{0}} f_{1} \ldots \widetilde{T}_{t_{n+m}-t_{n+m-1}} f_{n+m}\right)_{L^{2}\left(\mathbb{R}^{d}, \varphi_{0}^{2} \mathrm{~d} x\right)} .
\end{aligned}
$$

This implies the required time-shift invariance. Formula (2.10) is now a direct consequence, and this completes the proof of the theorem.

\section{Local path regularity of GST processes}

\subsection{Stochastic differential equation with jumps associated with the GST process}

The generator $\widetilde{L}=-\widetilde{H}$ of the ground state-transformed process $\left(\widetilde{X}_{t}\right)_{t \geq 0}$ can be determined explicitly yielding (1.3), which has first appeared in [23]. Since $H$ and $\widetilde{H}$ are unitary equivalent by (2.8), we have $\operatorname{Dom}(\widetilde{H})=U \operatorname{Dom}(H)$, and since $H$ is closed, also $\widetilde{H}$ is a closed operator. Moreover, since $H$ is self-adjoint, $\widetilde{H}$ is also self-adjoint with core $C_{\mathrm{c}}^{\infty}\left(\mathbb{R}^{d}\right)$.

To study the multifractal spectrum, first we show the existence of a solution to the martingale problem for $\left(\widetilde{L}, C_{\mathrm{c}}^{2}\left(\mathbb{R}^{d}\right)\right)$, and provide a jump SDE representation for the ground state-transformed process, which we call the ground state $S D E$. We write $\mathbb{R}_{*}^{d}=\mathbb{R}^{d} \backslash\{0\}$ for a shorthand notation.

For the ground state SDE we will use the following condition (see also Remark 3.1 below).

Assumption 3.1. Let $\varphi_{0}$ be the ground state of $H$. We assume that the function $x \mapsto \nabla \ln \varphi_{0}(x)$, $x \in \mathbb{R}^{d}$, is locally bounded. 
Theorem 3.1. Let Assumptions 2.1 and 3.1 hold. Consider the stochastic differential equation with jumps

$$
\begin{aligned}
& M_{t}=M_{0}+\sigma B_{t}+\int_{0}^{t} \sigma \nabla \ln \varphi_{0}\left(M_{s}\right) \mathrm{d} s+\int_{0}^{t} \int_{|z| \leq 1} \frac{\varphi_{0}\left(M_{s}+z\right)-\varphi_{0}\left(M_{s}\right)}{\varphi_{0}\left(M_{s}\right)} z \nu(z) \mathrm{d} z \mathrm{~d} s \\
& \quad+\int_{0}^{t} \int_{|z| \leq 1} \int_{0}^{\infty} z \mathbf{1}_{\left\{v \leq \frac{\varphi_{0}\left(M_{s-}+z\right)}{\varphi_{0}\left(M_{s-}\right)}\right\}} \tilde{N}(\mathrm{~d} s, \mathrm{~d} z, \mathrm{~d} v)+\int_{0}^{t} \int_{|z|>1} \int_{0}^{\infty} z \mathbf{1}_{\left\{v \leq \frac{\varphi_{0}\left(M_{s-}+z\right)}{\varphi_{0}\left(M_{s-}\right)}\right\}} N(\mathrm{~d} s, \mathrm{~d} z, \mathrm{~d} v),
\end{aligned}
$$

where $\left(B_{t}\right)_{t \geq 0}$ is an $\mathbb{R}^{d}$-valued Brownian motion with covariance matrix $\sigma$, and $N$ is a Poisson random measure on $[0, \infty) \times \mathbb{R}_{*}^{d} \times[0, \infty)$ with intensity $\mathrm{d} t \nu(z) \mathrm{d} z \mathrm{~d} v$. The GST process constructed in Theorem 2.1 is a weak solution of (3.1).

Proof. Let

$$
\widetilde{X}_{t}^{f}=f\left(\widetilde{X}_{t}\right)-f\left(\widetilde{X}_{0}\right)-\int_{0}^{t} \widetilde{L} f\left(\widetilde{X}_{r}\right) \mathrm{d} r, \quad t \geq 0
$$

where $f \in \operatorname{Dom}(\widetilde{L})$. Using a general result, see e.g. Kurtz [30, Th. 2.3], we have that $\left(\widetilde{X}, \widetilde{\mathbb{P}^{x}}\right)$ is a weak solution of the $\operatorname{SDE}(3.1)$ if and only if $\widetilde{\mathbb{P}}^{x}$ solves the $\left(\widetilde{L}, C_{\mathrm{c}}^{2}\right)$ martingale problem with initial value $x \in \mathbb{R}^{d}$, that is, $\left(\widetilde{X}_{t}^{f}\right)_{t \geq 0}$ is a martingale under $\widetilde{\mathbb{P}}^{x}$, for all $f \in C_{\mathrm{c}}^{2}\left(\mathbb{R}^{d}\right)$.

Using Assumption 3.1 and that $\varphi_{0}>0$ is bounded continuous, we see that the functions $x \mapsto$ $\int_{\mathbb{R}^{d}} \varphi_{0}(z+x) / \varphi_{0}(z)\left(1 \wedge|z|^{2}\right) \nu(\mathrm{d} z), x \mapsto \int_{|z| \leq 1} z\left(\varphi_{0}(z+x)-\varphi_{0}(z)\right) / \varphi_{0}(z) \nu(\mathrm{d} z)$ and $x \mapsto \nabla \log \varphi_{0}(x)$ are locally bounded, and the conditions in [30, Th. 2.3] hold. Also, since $\widetilde{L}$ is a closed operator, by using a mollifier we can close $C_{\mathrm{c}}^{\infty}\left(\mathbb{R}^{d}\right)$ in the $C^{2}$-norm as in [8, Th. 2.37] to obtain that $C_{\mathrm{c}}^{2}\left(\mathbb{R}^{d}\right) \subset$ $\operatorname{Dom}(\widetilde{L})$.

Let $\left(M, P^{x}\right)$ be a weak solution to (3.1) on a suitable probability space with probability measure $P^{x}$, and starting point $P^{x}\left(M_{0}=x\right)=1$. Write for the drift $b: \mathbb{R}^{d} \rightarrow \mathbb{R}^{d}$,

$$
b(x)=\sigma \nabla \ln \varphi_{0}(x)+\int_{|z| \leq 1} \frac{\varphi_{0}(x+z)-\varphi_{0}(x)}{\varphi_{0}(x)} z \nu(z) \mathrm{d} z .
$$

Using Itô's formula for $\mathbb{R}^{d}$-valued semimartingales, see e.g. [17], for every real-valued $f \in C_{\mathrm{c}}^{2}\left(\mathbb{R}^{d}\right)$ we have

$$
\begin{aligned}
& f\left(M_{t}\right)= f\left(M_{0}\right)+\int_{0}^{t} \nabla f\left(M_{s-}\right) \cdot \mathrm{d}\left(\sigma B_{s}\right)+\int_{0}^{t} \nabla f\left(M_{s}\right) \cdot b\left(M_{s}\right) \mathrm{d} s+\frac{1}{2} \int_{0}^{t} \sigma \nabla \cdot \sigma \nabla f\left(M_{s}\right) \mathrm{d} s \\
&+\int_{0}^{t} \int_{\mathbb{R}_{*}^{d}} \int_{0}^{\infty}\left[f \left(M_{s-}+z \mathbf{1}_{\left.\left.\left\{v \leq \frac{\varphi_{0}\left(M_{s-}+z\right)}{\varphi_{0}\left(M_{s-}\right)}\right\}\right)-f\left(M_{s-}\right)\right] \tilde{N}(\mathrm{~d} s, \mathrm{~d} z, \mathrm{~d} v)}\right.\right. \\
&+\int_{0}^{t} \int_{|z|>1} \int_{0}^{\infty}\left[f\left(M_{s-}+z \mathbf{1}_{\left\{v \leq \frac{\varphi_{0}\left(M_{s-}+z\right)}{\varphi_{0}\left(M_{s-}\right)}\right\}}\right)-f\left(M_{s-}\right)\right] \mathrm{d} v \nu(z) \mathrm{d} z \mathrm{~d} s \\
&+\int_{0}^{t} \int_{|z| \leq 1} \int_{0}^{\infty}\left[f\left(M_{s-}+z \mathbf{1}_{\left\{v \leq \frac{\varphi_{0}\left(M_{s-}+z\right)}{\varphi_{0}\left(M_{s-}\right)}\right\}}\right)-f\left(M_{s-}\right)\right. \\
&\left.-\nabla f\left(M_{s-}\right) \cdot z \mathbf{1}_{\left\{v \leq \frac{\varphi_{0}\left(M_{s-}+z\right)}{\varphi_{0}\left(M_{s-}\right)}\right\}}\right] \mathrm{d} v \nu(z) \mathrm{d} z \mathrm{~d} s .
\end{aligned}
$$

Note that in the second-to-last integral the integrand is zero for all $v$ larger than the ground state ratio $\varphi_{0}\left(M_{s-}+z\right) / \varphi_{0}\left(M_{s-}\right)$. It is thus equal to

$$
\int_{0}^{t} \int_{|z|>1}\left(f\left(M_{s-}+z\right)-f\left(M_{s-}\right)\right) \frac{\varphi_{0}\left(M_{s-}+z\right)}{\varphi_{0}\left(M_{s-}\right)} \nu(z) \mathrm{d} z \mathrm{~d} s
$$


Similarly, the last integral equals

$$
\int_{0}^{t} \int_{|z| \leq 1}\left(f\left(M_{s-}+z\right)-f\left(M_{s-}\right)-z \cdot \nabla f\left(M_{s-}\right)\right) \frac{\varphi_{0}\left(M_{s-}+z\right)}{\varphi_{0}\left(M_{s-}\right)} \nu(z) \mathrm{d} z \mathrm{~d} s .
$$

Since $f$ has bounded first and second derivatives, the Brownian component and the compensated Poisson integral are martingales, therefore

$$
M_{t}^{f}=f\left(M_{t}\right)-f\left(M_{0}\right)-\int_{0}^{t} \widetilde{L} f\left(M_{s}\right) \mathrm{d} s, \quad t \geq 0,
$$

is a $P^{x}$-martingale.

It remains to show that the probability measures $P^{x}=\widetilde{\mathbb{P}}^{x}$ constructed in Theorem 2.1 solve the $\left(\widetilde{L}, C_{\mathrm{c}}^{2}\right)$ martingale problem with initial value $x \in \mathbb{R}^{d}$. Consider the natural filtration $\left(\mathcal{F}_{t}\right)_{t \geq 0}$ of $\left(\widetilde{X}_{t}^{f}\right)_{t \geq 0}$ and let $0 \leq s \leq t$. Since $\mathbb{E}_{\widetilde{\mathbb{P} x}}\left[\widetilde{X}_{t}^{f} \mid \mathcal{F}_{s}\right]=\widetilde{X}_{s}^{f}+\mathbb{E}_{\widetilde{\mathbb{P} x}}\left[\widetilde{X}_{t}^{f}-\widetilde{X}_{s}^{f} \mid \mathcal{F}_{s}\right]$, we only need to show that the second term vanishes. By the Markov property of $\left(\widetilde{X}_{t}\right)_{t \geq 0}$ established in Theorem 2.1, we have

$$
\mathbb{E}_{\widetilde{\mathbb{P}}^{x}}\left[f\left(\widetilde{X}_{t}\right) \mid \mathcal{F}_{s}\right]=\widetilde{T}_{t-s} f\left(\widetilde{X}_{s}\right), \quad 0 \leq s \leq t .
$$

By differentiability of the function $t \mapsto \widetilde{T}_{t}$, we obtain for all $t \geq 0$ that $\frac{d}{d t} \widetilde{T}_{t} f=\widetilde{L}_{t} f=\widetilde{T}_{t} \widetilde{L} f$, and hence $\widetilde{T}_{t} f-f=\int_{0}^{t} \widetilde{L}_{r} f \mathrm{~d} r$. Thus we have

$$
\begin{aligned}
\mathbb{E}_{\widetilde{\mathbb{P}} x}\left[f\left(\widetilde{X}_{t}\right)-f\left(\widetilde{X}_{s}\right)-\int_{s}^{t} \widetilde{L} f\left(\widetilde{X}_{r}\right) \mathrm{d} r \mid \mathcal{F}_{s}\right] & =\widetilde{T}_{t-s} f\left(\widetilde{X}_{s}\right)-f\left(\widetilde{X}_{s}\right)-\int_{s}^{t} \widetilde{L} \widetilde{T}_{r-s} f\left(\widetilde{X}_{s}\right) \mathrm{d} r \\
& =\widetilde{T}_{t-s} f\left(\widetilde{X}_{s}\right)-f\left(\widetilde{X}_{s}\right)-\int_{0}^{t-s} \widetilde{L} \widetilde{T}_{r} f\left(\widetilde{X}_{s}\right) \mathrm{d} r \\
& =\widetilde{T}_{t-s} f\left(\widetilde{X}_{s}\right)-f\left(\widetilde{X}_{s}\right)-\widetilde{T}_{t-s} f\left(\widetilde{X}_{s}\right)+f\left(\widetilde{X}_{s}\right)=0,
\end{aligned}
$$

as required.

\section{Remark 3.1.}

(1) In general, little information is available on the regularity of $\varphi_{0}$. In some specific cases of potentials growing to infinity at infinity and the operator $L=\left(-\mathrm{d}^{2} / \mathrm{d} x^{2}\right)^{1 / 2}$, it is known that the ground state is analytic [33, 11]. However, it is also known that the ground state for Brownian motion in a finitely deep potential well, i.e., $V(x)=-v \mathbf{1}_{\{|x| \leq a\}}, v, a>0$, is only $C^{1}$.

(2) The conditions under which the ground state SDE has a solution and Theorem 3.1 holds can be improved. For a large class of Lévy processes $\left(X_{t}\right)_{t \geq 0}$ and potentials $V$, it can be shown that for large enough $|x|$ and suitable constants $C_{1}, C_{2}>0$,

$$
\begin{array}{ll}
C_{1} \frac{\nu(x)}{V(x)} \leq \varphi_{0}(x) \leq C_{2} \frac{\nu(x)}{V(x)} & \text { for } V(x) \rightarrow \infty \text { as }|x| \rightarrow \infty \\
C_{1} \nu(x) \leq \varphi_{0}(x) \leq C_{2} \nu(x) \quad \text { for } V(x) \rightarrow 0 \text { as } \quad|x| \rightarrow \infty .
\end{array}
$$

For precise statements and conditions we refer to [22, 24]. For illustration consider $d=1, V(x) \asymp$ $x^{2 m}, m>0$, and a symmetric $\alpha$-stable process; then the above implies $\varphi_{0}(x) \asymp|x|^{-d-\alpha-2 m}$, and thus far enough from the origin the drift would become $b(x)=\sigma \frac{\mathrm{d}}{\mathrm{d} x} \ln \varphi_{0}(x) \asymp-\frac{\operatorname{sgn}(x)}{|x|}$. Hence for large enough values of $X_{t}$ we get $X_{t} b\left(X_{t}\right)<0$, and this pull-back mechanism would prevent the paths from exploding. Since our main concern here is the multifractal behaviour of GST processes, the ground state SDE will be studied in further detail elsewhere.

(3) We note that we are not concerned with uniqueness of the solution of the martingale problem. Since we show below that any solution of the SDE (3.1) has the same multifractal nature, we only need to know that the GST process is a solution. 


\subsection{Multifractal spectrum of GST processes}

Now we are in the position to state and prove the multifractal nature of local Hölder exponents of ground state-transformed processes via the ground state SDE. Recall the notations in (1.7)-(1.8).

Theorem 3.2. Let Assumptions 2.1 and 3.1 hold, and $\left(M, \mathbb{P}^{x}\right)$ be a weak solution of (3.1).

(1) If $\sigma \neq 0$, i.e., the underlying Lévy process has a Brownian component, then almost surely

$$
D_{M}(h)=D_{X}^{1}(h), \quad h>0 .
$$

(2) If $\sigma=0$, i.e., the underlying Lévy process is a pure jump process, and

(i) either $\beta_{\nu} \in[1,2]$,

(ii) or $\beta_{\nu} \in(0,1)$ with $\varphi_{0} \in C^{k+1}\left(\mathbb{R}^{d}\right)$ and $k \geq 1 / \beta_{\nu}$,

then almost surely

$$
D_{M}(h)=D_{X}^{2}(h), \quad h>0 .
$$

In the remainder of this section we prove this theorem through a sequence of auxiliary results.

\subsubsection{Associated Poisson point process}

Recall that the jumps of the Poisson measure $N$ give rise to a Poisson point process with measure $\nu(z) \mathrm{d} z$. The pointwise regularity of Lévy processes with infinite jump measure, i.e., $\int_{\mathbb{R}^{d}} \nu(z) \mathrm{d} z=\infty$, relies on a configuration of a dense set of jumps. For the process $\left(M_{t}\right)_{t \geq 0}$ the jump configuration is more involved as it depends on the entire paths of the process. Indeed, the factor featuring the indicator function in the compensated Poisson integral in (3.1) implies that our process jumps only as long as the ground state ratio is not too small. Thus $\left(M_{t}\right)_{t \geq 0}$ jumps less often than the underlying Lévy process. We prove that the underlying Poisson point process characterizes the pointwise regularity of $\left(M_{t}\right)_{t \geq 0}$.

Let $N(\mathrm{~d} t, \mathrm{~d} z, \mathrm{~d} v)$ be a Poisson measure with intensity $\mathrm{d} t n(\mathrm{~d} z, \mathrm{~d} v)$ on $\mathbb{R}^{+} \times E$ with $E=\mathbb{R}_{*}^{d} \times(0, \infty)$, endowed with the product Borel $\sigma$-field $\mathcal{B}(E)$. Let $\left\{E_{k}, k \in \mathbb{N}_{*}\right\}$ be a partition of $E$, with $E_{k} \in \mathcal{B}(E)$ and $n\left(E_{k}\right)<\infty$. It is well-known [15, Ths 8.1,9.1] that there exists

- a sequence of exponential random variables $\left\{\tau_{i}^{(k)}, i \in \mathbb{N}\right\}$ with parameter $n\left(E_{k}\right)$,

- a sequence of random variables $\left\{\xi_{i}^{(k)}, i \in \mathbb{N}\right\}$ with distribution $\mathbf{1}_{E_{k}} n(\mathrm{~d} z, \mathrm{~d} v) / n\left(E_{k}\right)$,

such that

$$
N((0, t] \times U)=|\{s \in D: s \leq t, p(s) \in U\}|, \text { for all } t>0, U \in \mathcal{B}(E),
$$

where $p$ is the point process defined by

$$
p\left(\sum_{\ell=0}^{i} \tau_{\ell}^{(k)}\right)=\xi_{i}^{(k)}, \quad k, i=1,2, \ldots
$$

and

$$
D=\bigcup_{k=0}^{\infty}\left\{\sum_{\ell=0}^{i} \tau_{\ell}^{(k)}: i \in \mathbb{N}\right\} .
$$

Here, all $\tau_{i}^{(k)}, \xi_{i}^{(k)}$ are mutually independent random variables on the same probability space. Extending the probability space, if necessary, by passing to a product probability space, we can find a 
sequence of uniform random variables $\eta_{i}$ in $[0,1]$ that is independent of $p$. Define $p^{\prime}: D \rightarrow E \times[0,1]$ by

$$
p^{\prime}\left(\sum_{\ell=0}^{i} \tau_{\ell}^{(k)}\right)=\left(\xi_{i}^{(k)}, \eta_{i}\right) \quad k, i=1,2, \ldots
$$

It can be shown $[15$, Ths $8.1,9.1]$ that the counting measure

$$
N_{p^{\prime}}((0, t] \times U \times I)=\left|\left\{s \in D: s \leq t, p^{\prime}(s) \in U \times I\right\}\right|, \text { for all } t>0, U \in \mathcal{B}(E), I \in \mathcal{B}([0,1]),
$$

is also a Poisson measure, with intensity $\mathrm{d} t n(\mathrm{~d} z, \mathrm{~d} v) \mathbf{1}_{[0,1]}(x) \mathrm{d} x$. In particular, almost surely,

$$
N((0, t] \times U)=N_{p^{\prime}}((0, t] \times U \times[0,1]) .
$$

From now on, we consider the Poisson measure $N$ as part of the weak solution $\left(M, \widetilde{\mathbb{P}}^{x}\right)$ of the SDE (3.1) on a probability space. Possibly on the extended probability space, we have $N_{p^{\prime}}$ satisfying (3.3) which will serve as an auxiliary measure to prove a covering property satisfied by a family of point systems induced by the jumps of our process $\left(M_{t}\right)_{t \geq 0}$. Informally, the measure $N_{p^{\prime}}$ allows us to remove spatial dependence of the jump kernel; a similar argument has been first used in [42]. In what follows we will write

$$
p=\{(s, z(s), v(s)): s \in D\} \quad \text { and } \quad p^{\prime}=\{(s, z(s), v(s), x(s)): s \in D\} .
$$

\subsubsection{Hölder regularity}

First we determine the pointwise Hölder exponent of the sample paths of the ground state SDE under the assumption that the ratio of ground state evaluations appearing in the coefficients of the SDE is bounded both from below and above, i.e., we assume that there exists $0<c<1$ such that

$$
c \leq \frac{\varphi_{0}(x+z)}{\varphi_{0}(x)} \leq 1 / c, \quad x \in \mathbb{R}^{d},|z| \leq 1 .
$$

In a next step we remove this constraint by using a localization argument to get the result in a desirable generality.

The following general result is due to Jaffard [19, Lem. 1], which is essential in deriving an upper bound for the Hölder exponent of a locally bounded function with a dense set of jump discontinuities.

Lemma 3.1. Let $f: \mathbb{R} \rightarrow \mathbb{R}^{d}$ be a càdlàg function having a dense set of jump discontinuities of size $z_{n}$ at the time-points $t_{n}$. Then, for every $t \in \mathbb{R}$ and every sequence of jump discontinuities $t_{n_{k}} \rightarrow t$ as $k \rightarrow \infty$, we have

$$
H_{f}(t) \leq \liminf _{k \rightarrow \infty} \frac{\ln z_{n_{k}}}{\ln \left|t-t_{n_{k}}\right|} .
$$

Clearly, only the small jumps have an impact on the local regularity. Write

$$
J=\left\{s \geq 0:|z(s)| \leq 1, v(s) \leq \frac{\varphi_{0}\left(M_{s-}+z(s)\right)}{\varphi_{0}\left(M_{s-}\right)}\right\} .
$$

By the properties of the (compensated) Poisson integral, the solution to the ground state SDE (3.1) makes a jump at each $s \in J$, of size $|z(s)|$. Borrowing an idea from [19], we consider a family of limsup sets built from the Poisson point process $p$. Recall that $\beta_{\nu}$ is the Blumenthal-Getoor index of the Lévy measure $\nu(z) \mathrm{d} z$ defined in (1.6). For all $\delta>0$, define

$$
A(\varepsilon, \delta)=\bigcup_{s \in J,|z(s)| \leq \varepsilon}\left(s-|z(s)|^{\beta_{\nu} \delta}, s+|z(s)|^{\beta_{\nu} \delta}\right),
$$


and

$$
A(\delta)=\limsup _{\varepsilon \downarrow 0} A(\varepsilon, \delta) .
$$

This family of sets satisfies a convenient covering property when $\delta<1$.

Lemma 3.2. For all $\delta<1$ we have $A_{\delta}=[0, \infty)$, almost surely.

Proof. Define

$$
J^{\prime}=\left\{s \geq 0:|z(s)| \leq 1, v(s) \leq \frac{\varphi_{0}\left(M_{s-}+z(s)\right)}{\varphi_{0}\left(M_{s-}\right)}, x(s) \leq \frac{c \varphi_{0}\left(M_{s-}\right)}{\varphi_{0}\left(M_{s-}+z(s)\right)}\right\} \subset J,
$$

and $A_{\delta}^{\prime}$ as in (3.5) with $J$ replaced by $J^{\prime}$. Observe that by the lower bound in (3.4), the right hand side of the bound concerning $x(s)$ in the above set is a random number in $[0,1]$. Since $A_{\delta}^{\prime} \subset A_{\delta}$ for all $\delta \geq 0$, it remains to show that for any fixed $\delta<1$, we have $A_{\delta}^{\prime}=[0, \infty)$ almost surely. The result then follows by the monotonicity of the sets $A_{\delta}$ in $\delta$.

Step 1: First we note that the counting measure

$$
\mu(\mathrm{d} s, \mathrm{~d} y)=\sum_{s \in J} \delta_{\left(s,\left|z_{s}\right|{ }^{\delta \beta_{\nu}}\right)}
$$

is a Poisson random measure with intensity $\mathrm{d} s\left(c \pi_{\delta}(\mathrm{d} y)\right)$ on $\mathbb{R}^{+} \times(0,1]$, where $\pi_{\delta}$ is the image measure of $\nu(z) \mathrm{d} z 1_{|z| \leq 1}$ by the map $z \mapsto|z|^{\delta \beta_{\nu}}$ and $c$ is the constant in (3.4). For any predictable non-negative process $(s, y) \mapsto H(s, y)$,

$$
\begin{aligned}
& \int_{0}^{t} \int_{0}^{1} H(s, y) \mu(\mathrm{d} s, \mathrm{~d} y)-\int_{0}^{t} \int_{|z| \leq 1} H\left(s,|z(s)|^{\delta \beta_{\nu}}\right) c \nu(z) \mathrm{d} z \mathrm{~d} s \\
& =\int_{0}^{t} \int_{|z| \leq 1} \int_{0}^{\infty} \int_{0}^{1} \mathbf{1}_{\left\{v(s) \leq \frac{\varphi_{0}\left(M_{s-}+z(s)\right)}{\varphi_{0}\left(M_{s-}\right)}, x(s) \leq \frac{c \varphi_{0}\left(M_{s-}\right)}{\varphi_{0}\left(M_{s-}+z(s)\right)}\right\}} H\left(s,|z(s)|^{\delta \beta_{\nu}}\right) \tilde{N}_{p^{\prime}}(\mathrm{d} s, \mathrm{~d} z, \mathrm{~d} v, \mathrm{~d} x)
\end{aligned}
$$

is a local martingale. Then the compensator of $\mu$ is $c \mathrm{~d} t \pi_{\delta}(\mathrm{d} y)$. By [17, Ch.2, Th.1.8], $\mu$ is a Poisson measure with intensity $c \mathrm{~d} t \pi_{\delta}(\mathrm{d} y)$.

Step 2: Applying the integral test of covering for limsup sets built from a Poisson measure, see $[4,39]$, we only need to show that

$$
\int_{0}^{1} \exp \left(2 \int_{t}^{1} c \pi_{\delta}((y, 1)) \mathrm{d} y\right) \mathrm{d} t=\infty .
$$

The divergence of this integral can be proved by a modification of $[19$, Lem. 2$]$. Note that

$$
\int_{t}^{1} c \pi_{\delta}((y, 1)) \mathrm{d} y=c \int_{t^{\frac{1}{\delta \beta_{\nu}}}}^{1}\left(\int_{u<|x|<1} \nu(x) \mathrm{d} x\right) \delta \beta_{\nu} u^{\delta \beta_{\nu}-1} \mathrm{~d} u .
$$

Write $C_{j}=\int_{2^{-j-1}<|x| \leq 2^{-j}} \nu(x) \mathrm{d} x$ and $\omega(u)=\int_{u<|x|<1} \nu(x) \mathrm{d} x$. Let $j(t)$ be the unique integer such that $2^{j(t)-3}<t^{\frac{1}{\delta \beta_{\nu}}} \leq 2^{-j(t)-2}$. Then we have

$$
\begin{aligned}
\int_{t^{\frac{1}{\delta \beta_{\nu}}}}^{1} \omega(u) \delta \beta_{\nu} u^{\delta \beta_{\nu}-1} \mathrm{~d} u & \geq \int_{2^{j(t)-2}}^{2^{j(t)-1}} \omega(u) \delta \beta_{\nu} u^{\delta \beta_{\nu}-1} \mathrm{~d} u \\
& \geq C_{j(t)} \delta \beta_{\nu}\left(2^{-j(t)-2}\right)^{\delta \beta_{\nu}-1} 2^{-j(t)-2} \\
& =C_{j(t)} \delta \beta_{\nu}\left(2^{-j(t)-2}\right)^{\delta \beta_{\nu}}
\end{aligned}
$$


By the definition of $\beta_{\nu}$, for any $r \in\left(\delta \beta_{\nu}, \beta_{\nu}\right)$, there exist infinitely many $j$ such that $C_{j} \geq 2^{r j}$. For any such $j$ we have

$$
\int_{2^{-(j+3) \delta \beta_{\nu}}}^{2^{-(j+2) \delta \beta_{\nu}}} \exp \left(2 \int_{t}^{1} c \pi_{\delta}((y, 1)) \mathrm{d} y\right) \mathrm{d} t \geq\left(2^{\delta \beta_{\nu}}-1\right) 2^{-(j+3) \delta \beta_{\nu}} \exp \left(c \delta \beta_{\nu} 2^{1-2 \delta \beta_{\nu}} 2^{j(r-\delta \beta)}\right),
$$

which is bounded from below by 1 for all $j$ sufficiently large.

The latter lemma is a uniform approximation property of every time by the jumps. It is clear that $A_{\delta}$ is monotone in $\delta$, while the critical value is $\delta=1$ for which the limsup set may or may not cover the semi-axis. As soon as $\delta<1$, full covering occurs. In particular, whenever $\delta<1$, for every $t \geq 0$ there exist infinitely many $s_{n} \in J$ with $\left|z\left(s_{n}\right)\right| \downarrow 0$, such that

$$
\left|t-s_{n}\right| \leq\left|z\left(s_{n}\right)\right|^{\beta_{\nu} \delta} \text {. }
$$

For fixed time-points, one might expect an improved inequality to hold, which motivates the notion of the pointwise approximation rate defined below.

Definition 3.1. Let $\left(t_{n}, r_{n}\right) \in \mathbb{R}^{+} \times \mathbb{R}_{*}$ be a family of points. We call

$$
\delta_{t}=\sup \left\{\delta \geq 0:\left|t-t_{n}\right| \leq r_{n}^{\beta_{\nu} \delta} \text { infinitely often }\right\}
$$

the approximation rate of $t \in \mathbb{R}^{+}$by the family of points.

The approximation rate is crucial in investigating the pointwise Hölder exponent of jump processes. By the covering lemma, for all $t \geq 0$ we have $\delta_{t} \geq 1$, almost surely. The use of this concept will appear clearly in the upper estimate of $H_{M}(t)$ below.

Proposition 3.1. For all $t \geq 0$,

$$
H_{M}(t) \leq \frac{1}{\beta_{\nu} \delta_{t}}
$$

almost surely.

Proof. Take any $t \in A_{\delta}$. An application of Lemma 3.1 to $\left(M_{t}\right)_{t \geq 0}$ and the set of $s_{n}$ in (3.6) implies that $H_{M}(t) \leq 1 /\left(\beta_{\nu} \delta\right)$. For an arbitrary $t$, we have the following cases. If $\delta_{t}<\infty$, then for any $\varepsilon>0, t \in A_{\delta_{t}-\varepsilon}$, we have $H_{M}(t) \leq 1 /\left(\beta_{\nu}\left(\delta_{t}-\varepsilon\right)\right)$. Letting $\varepsilon \rightarrow 0$ gives the result. If $\delta_{t}=\infty$, then $t \in \cap_{\delta \geq 1} A_{\delta}$, and thus $H_{M}(t)=0$, which is the claimed upper bound.

To derive a lower bound, we need to control the increments of the sample paths. An analogue of the following result appears in [1] for Lévy processes, however, since the GST processes have position-dependent increments, we need a substantial upgrading. For each $n \in \mathbb{N}$, write

$$
Y_{n}(t)=\int_{0}^{t} \int_{|z| \leq 2^{-\frac{n}{\delta \beta_{\nu}}}} \int_{0}^{\infty} \mathbf{1}_{\left\{v \leq \frac{\varphi_{0}\left(M_{s-}+z\right)}{\varphi_{0}\left(M_{s-}\right)}\right\}} z \tilde{N}(\mathrm{~d} s, \mathrm{~d} z, \mathrm{~d} v)
$$

Lemma 3.3. Let $\delta>1$. There exist finite constants $c_{1}, c_{2}>0$ such that for all $n \in \mathbb{N}$,

$$
\mathbb{P}\left(\sup _{s, t \in[0,1],|s-t| \leq 2^{-n}}\left|Y_{n}(t)-Y_{n}(s)\right| \geq 3 n 2^{-\frac{n}{\delta \beta_{\nu}}} \sqrt{d}\right) \leq c_{1} e^{-c_{2} n} .
$$

Proof. Let $I_{n, k}=\left[k 2^{-n},(k+1) 2^{-n}\right)$. Using a dyadic approximation, the required probability can be bounded from above by

$$
\sum_{k=0}^{2^{n}-1} \mathbb{P}\left(\sup _{t \in I_{n, k}}\left|Y_{n}(t)-Y_{n}\left(k 2^{-n}\right)\right| \geq n 2^{-\frac{n}{\delta \beta_{\nu}}} \sqrt{d}\right)
$$


We estimate the sum term by term. For each $k$, consider the semimartingale

$$
\widetilde{Y}_{n}(t)=2^{\frac{n}{\delta \beta_{\nu}}}\left(Y_{n}\left(t+k 2^{-n}\right)-Y_{n}\left(k 2^{-n}\right), \quad t \in I_{n, k} .\right.
$$

Applying Itô's formula with the map $x \mapsto e^{x \cdot \xi}$, where $\xi=\overrightarrow{e_{i}}$ is the canonical orthonormal basis of $\mathbb{R}^{d}$, we obtain

$$
\begin{aligned}
e^{\widetilde{Y}_{n}(t) \cdot \xi}=1+ & \int_{k 2^{-n}}^{t} \int_{|z| \leq 2}-\frac{n}{\delta \beta_{\nu}} \int_{0}^{\infty} e^{\widetilde{Y}_{n}(s-) \cdot \xi}\left(\exp \left(2^{\frac{n}{\delta \beta_{\nu}}} \mathbf{1}_{\left\{v \leq \frac{\varphi_{0}\left(M_{s-}+z\right)}{\varphi_{0}\left(M_{s-}\right)}\right\}} z \cdot \xi\right)-1\right) \tilde{N}(\mathrm{~d} s, \mathrm{~d} z, \mathrm{~d} v) \\
& +\int_{k 2^{-n}}^{t} \int_{|z| \leq 2^{-\frac{n}{\delta \beta_{\nu}}}} \int_{0}^{\infty} e^{\widetilde{Y}_{n}(s-) \cdot \xi}\left(\exp \left(2^{\frac{n}{\delta \beta_{\nu}}} \mathbf{1}_{\left\{v \leq \frac{\varphi_{0}\left(M_{s-}+z\right)}{\varphi_{0}\left(M_{s-}\right)}\right\}} z \cdot \xi\right)-1\right. \\
& \left.-2^{\frac{n}{\delta \beta_{\nu}}} \mathbf{1}_{\left\{v \leq \frac{\varphi_{0}\left(M_{s-}+z\right)}{\varphi_{0}\left(M_{s-}\right)}\right\}} z \cdot \xi\right) \mathrm{d} v \nu(z) \mathrm{d} z \mathrm{~d} s,
\end{aligned}
$$

for all $t \in I_{n, k}$. Define the stopping times $\tau_{r}=\inf \left\{t \in I_{n, k}:\left|\widetilde{Y}_{n}(t)\right| \geq r\right\}, r \in \mathbb{N}$, with the convention that $\inf \emptyset=\infty$. By the càdlàg property of sample paths, $\tau_{r} \rightarrow \infty$ as $r \rightarrow \infty$, almost surely. Since the stopped compensated Poisson integral is a centered martingale, on taking expectation in the above formula and using $\left|e^{u}-1-u\right| \leq u^{2}$ for $|u| \leq 1$, we get

$$
\begin{aligned}
\mathbb{E}\left[e^{\widetilde{Y}_{n}\left(t \wedge \tau_{r}\right) \cdot \xi}\right] & \leq 1+\mathbb{E}\left[\int_{k 2^{-n}}^{t \wedge \tau_{r}} \int_{|z| \leq 2^{-\frac{n}{\delta \beta_{\nu}}}} \int_{0}^{\infty} e^{\widetilde{Y}_{n}(s-) \cdot \xi} \mathbf{1}_{\left\{v \leq \frac{\varphi_{0}\left(M_{s-}+z\right)}{\varphi_{0}\left(M_{s-}\right)}\right\}}\left(2^{\frac{n}{\delta \beta_{\nu}}} z \cdot \xi\right)^{2} \mathrm{~d} v \nu(z) \mathrm{d} z \mathrm{~d} s\right] \\
& =1+\mathbb{E}\left[\int_{k 2^{-n}}^{t \wedge \tau_{r}} \int_{|z| \leq 2^{-\frac{n}{\delta \beta_{\nu}}}} \frac{\varphi_{0}\left(M_{s-}+z\right)}{\varphi_{0}\left(M_{s-}\right)} e^{\widetilde{Y}_{n}(s-) \cdot \xi}\left(2^{\frac{n}{\delta \beta_{\nu}}} z \cdot \xi\right)^{2} \nu(z) \mathrm{d} z \mathrm{~d} s\right] .
\end{aligned}
$$

Using the upper bound in (3.4), we furthermore obtain

$$
\mathbb{E}\left[e^{\widetilde{Y}_{n}\left(t \wedge \tau_{r}\right) \cdot \xi}\right] \leq 1+\frac{1}{c} \mathbb{E}\left[\int_{k 2^{-n}}^{t \wedge \tau_{r}} e^{\widetilde{Y}_{n}(s-) \cdot \xi} \mathrm{d} s \int_{|z| \leq 2}{ }_{-\frac{n}{\delta \beta_{\nu}}} 2^{\frac{2 n}{\delta \beta_{\nu}}}|z|^{2-\delta \beta_{\nu}}|z|^{\delta \beta_{\nu}} \nu(z) \mathrm{d} z\right] .
$$

The integral over $z$ in the expectation is bounded above by

$$
2^{n} \int_{|z| \leq 1}|z|^{\delta \beta_{\nu}} \nu(z) \mathrm{d} z=C_{1} 2^{n}
$$

with a suitable constant $C_{1}$, which does not depend on $n$ and is finite since $\delta>1$. By Fubini's theorem,

$$
\mathbb{E}\left[e^{\widetilde{Y}_{n}\left(t \wedge \tau_{r}\right) \cdot \xi}\right] \leq 1+\frac{2^{n}}{c_{1}} \int_{k 2^{-n}}^{t} \mathbb{E}\left[e^{\widetilde{Y}_{n}\left(s \wedge \tau_{r}\right) \cdot \xi}\right] \mathrm{d} s,
$$

where $C_{2}=C_{1} / c$. Gronwall's lemma yields then

$$
\mathbb{E}\left[e^{\widetilde{Y}_{n}\left(t \wedge \tau_{r}\right) \cdot \xi}\right] \leq e^{\left(t-k 2^{-n}\right) 2^{n} / C_{2}} \leq e^{1 / C_{2}},
$$

for all $t \in I_{n, k}$. Letting $r \rightarrow \infty$ and using Fatou's lemma, we get $\mathbb{E}\left[e^{\widetilde{Y}_{n}(t) \cdot \xi}\right] \leq e^{1 / C_{2}}$, and similarly $\mathbb{E}\left[e^{-\widetilde{Y}_{n}(t) \cdot \xi}\right] \leq e^{1 / C_{2}}$. Hence, using that $x_{1}{ }^{2}+\cdots+x_{d}{ }^{2} \leq d \max _{i} x_{i}^{2}$, we have

$$
\mathbb{E}\left[e^{\left|\widetilde{Y}_{n}(t)\right| / \sqrt{d}}\right] \leq \sum_{i=1}^{d} \mathbb{E}\left[e^{\left|\widetilde{Y}_{n}(t) \cdot \vec{e}_{i}\right|}\right] \leq 2 d e^{1 / C_{2}}
$$

To conclude, by the Markov inequality we see that each term in (3.7) is bounded from above by $e^{-n} \mathbb{E}\left[e^{\left|\widetilde{Y}_{n}(t)\right| / \sqrt{d}}\right]$, which is summable in $n$.

Remark 3.2. This lemma can be extended to any bounded interval. We thus focus on the unit interval $[0,1]$. 
We can now prove a lower bound for the Hölder exponent of the compensated Poisson integral

$$
Y_{t}=\int_{0}^{t} \int_{|z| \leq 1} \int_{0}^{\infty} \mathbf{1}_{\left\{v \leq \frac{\varphi_{0}\left(M_{s-}+z\right)}{\varphi_{0}\left(M_{s-}\right)}\right\}} z \tilde{N}(\mathrm{~d} s, \mathrm{~d} z, \mathrm{~d} v) .
$$

Proposition 3.2. For all $t \in[0,1] \backslash J$,

$$
H_{Y}(t) \geq \frac{1}{\delta_{t} \beta_{\nu}}
$$

almost surely.

Proof. The Borel-Cantelli lemma combined with Lemma 3.3 give that for all $n$ larger than a suitable $n_{0} \in \mathbb{N}$,

$$
\sup _{s, t \in[0,1],|s-t| \leq 2^{-n}}\left|Y_{n}(t)-Y_{n}(s)\right| \leq 3 \sqrt{d} n 2^{-\frac{n}{\delta \beta_{\nu}}}, \quad \text { a.s. }
$$

Fix a point of continuity $t \in[0,1] \backslash A_{\delta}$. Let $s$ be close enough to $t$ so that for some $n<n_{0}$,

$$
2^{-n-1}<|t-s| \leq 2^{-n}
$$

Then

$$
\left|Y_{n}(t)-Y_{n}(s)\right| \leq 6 \sqrt{d} \log \left(\frac{1}{|s-t|}\right)|t-s|^{\frac{1}{\delta_{\nu}}}
$$

Enlarging the value of $n_{0}$ if necessary, we see that $t \notin A_{\delta}$ implies that any jump $s_{p} \in[s, t]$ satisfies

$$
2^{-n} \geq|s-t| \geq\left|s_{p}-t\right| \geq\left|z\left(s_{p}\right)\right|^{\delta \beta_{\nu}}
$$

i.e., there are no jumps at the time-points $s_{p} \in J \cap[s, t]$ of size $\left|z\left(s_{p}\right)\right| \geq 2^{-\frac{n}{\delta \beta_{\nu}}}$. Hence,

$$
\begin{aligned}
& \left|\int_{s}^{t} \int_{1 \geq|z| \geq 2^{-\frac{n}{\delta \beta_{\nu}}}} \int_{0}^{\infty} \mathbf{1}_{\left\{v \leq \frac{\varphi_{0}\left(M_{s-}+z\right)}{\varphi_{0}\left(M_{s-}\right)}\right\}} z \widetilde{N}(\mathrm{~d} s, \mathrm{~d} z, \mathrm{~d} v)\right| \\
& \quad=\left|\int_{s}^{t} \int_{1 \geq|z| \geq 2^{-\frac{n}{\delta \beta_{\nu}}}} \int_{0}^{\infty} \mathbf{1}_{\left\{v \leq \frac{\varphi_{0}\left(M_{s-}+z\right)}{\varphi_{0}\left(M_{s-}\right)}\right\}} z \mathrm{~d} v \nu(z) \mathrm{d} z \mathrm{~d} s\right| \\
& \quad \leq|s-t| \frac{\varphi_{0}\left(M_{s-}+z\right)}{\varphi_{0}\left(M_{s-}\right)} \int_{1 \geq|z| \geq 2^{-\frac{n}{\delta \beta_{\nu}}}}|z| \nu(z) \mathrm{d} z
\end{aligned}
$$

The integral over $z$ is bounded above by

$$
\left(2^{-\frac{n}{\delta \beta_{\nu}}}\right)^{1-\delta \beta_{\nu}} \int_{|z| \leq 1}|z|^{\delta \beta_{\nu}} \nu(z) \mathrm{d} z \leq C|s-t|^{\frac{1}{\delta \beta_{\nu}}-1},
$$

with a constant $C>0$. Combining these estimates, we get

$$
\begin{aligned}
\left|Y_{s}-Y_{t}\right| & \leq\left|Y_{n}(t)-Y_{n}(s)\right|+\left|\int_{s}^{t} \int_{1 \geq|z| \geq 2^{-\frac{n}{\delta \beta_{\nu}}}} \int_{0}^{\infty} \mathbf{1}_{\left\{v \leq \frac{\varphi_{0}\left(M_{s-}+z\right)}{\varphi_{0}\left(M_{s-}\right)}\right\}} z \tilde{N}(\mathrm{~d} s, \mathrm{~d} z, \mathrm{~d} v)\right| \\
& \leq c|t-s|^{\frac{1}{\delta \beta_{\nu}}} \log \frac{1}{|s-t|}
\end{aligned}
$$

where $c$ is a finite constant dependent on $M$ and $d$. Hence, almost surely, for all rational $\delta>1$ we have $H_{Y}(t) \geq 1 /\left(\delta \beta_{\nu}\right)$ at all times of continuity $t \in[0,1] \backslash A_{\delta}$. By the definition of $\delta_{t}$, it is seen that $H_{Y}(t) \geq 1 /\left(\delta_{t} \beta_{\nu}\right)$, for all continuity points $t \in[0,1]$, almost surely.

Remark 3.3. Using the argument in the proof of Proposition 3.1, we can similarly show $H_{Y}(t) \leq$ $1 /\left(\delta_{t} \beta_{\nu}\right)$ for all $t$. 
Theorem 3.3. Under the assumptions of Theorem 1.3, for all times of continuity $t$,

$$
H_{M}(t)= \begin{cases}\frac{1}{\delta_{t} \beta_{\nu}} \wedge \frac{1}{2} & \text { if } \sigma \neq 0 \\ \frac{1}{\delta_{t} \beta_{\nu}} & \text { if } \sigma=0\end{cases}
$$

almost surely.

Proof. We distinguish three situations according to the matrix $\sigma$ and the value of $\beta_{\nu}$.

Case 1: Let $\sigma \neq 0$ and $\beta_{\nu} \in(0,2]$. Recall that for any $f, g: \mathbb{R}^{+} \rightarrow \mathbb{R}^{d}$ locally bounded functions $H_{f+g}(t) \geq \min \left(H_{f}(t), H_{g}(t)\right.$, where equality holds when the Hölder exponents of $f$ and $g$ are different at $t$. Since the Hölder exponent of Brownian motion is $1 / 2$ everywhere and the drift terms are differentiable at every $t$ (necessarily their Hölder exponent is larger or equal to 1 ), we see that the sum of Brownian motion and the two drifts has Hölder exponent equal to $1 / 2$ everywhere. The uncompensated Poisson integral is locally constant, thus it does not influence the local regularity of $\left(M_{t}\right)_{t \geq 0}$ except on the set of jump times (finite in any bounded interval). The compensated Poisson integral has Hölder exponent $1 /\left(\delta_{t} \beta_{\nu}\right)$ at any point of continuity $t$. The claimed formula follows if $1 /\left(\delta_{t} \beta_{\nu}\right) \neq 1 / 2$, otherwise $1 / 2$ is a straightforward lower bound for $H_{M}(t)$, and it is also an upper bound due to Lemma 3.1. Thus the identity follows.

Case 2: Let $\sigma=0$ and $\beta_{\nu} \in[1,2]$. In this case, $1 /\left(\delta_{t} \beta_{\nu}\right) \leq 1$, since $\delta_{t} \geq 1$ for all $t$, i.e., the drifts are all smoother than the compensated Poisson integral. The result follows.

Case 3: Let $\sigma=0$ and $\beta_{\nu} \in(0,1)$. Our assumption implies that the drift terms are smoother than the compensated Poisson integral. To see this, note that for any locally bounded $f: \mathbb{R}^{+} \rightarrow \mathbb{R}^{d}$, $g: \mathbb{R}^{d} \rightarrow \mathbb{R}^{d}$, with $F(t)=\int_{0}^{t} g(f(s)) \mathrm{d} s$, we have that whenever $g \in C^{k}\left(\mathbb{R}^{d}\right)$ with $k \geq H_{f}(t)$, it follows that $H_{F}(t) \geq 1+H_{f}(t)$. In particular, we have $H_{F}(t)>H_{f}(t)$. Applying this to $f=M$ and with $g$ chosen to be the drift coefficient in (3.1), combined with the fact that $H_{M}(t) \leq 1 /\left(\delta_{t} \beta_{\nu}\right) \leq 1 / \beta_{\nu}$, yields $H_{M}(t)=H_{Y}(t)$, as claimed.

To complete the proof, in a concluding step we remove condition (3.4). Let

$$
\Omega_{K, b}=\left\{\omega \in \Omega: \sup _{t \leq b}\left|M_{t}(\omega)\right| \leq K\right\} .
$$

The càdlàg properties of the sample paths imply that $\mathbb{P}\left(\Omega_{K, b}\right) \rightarrow 1$ as $K \rightarrow \infty$. Assumption 2.1 implies that the two-sided inequality in (3.4) holds uniformly for $|z| \leq 1,|x| \leq K$, for every $K \in \mathbb{N}_{*}$, with $c$ dependent on the value of $K$. For paths in $\Omega_{K, b}$, we have shown the result above. Letting $K \rightarrow \infty$, then $b \rightarrow \infty$ completes the proof.

\subsubsection{Proof of Theorem 3.2: multifractal spectrum}

We determine the multifractal spectrum under condition (3.4); the extension to the general situation can be done as at the end of the last subsection.

Note that by Theorem 3.3 it suffices to consider $h \in\left[0,1 / \beta_{\nu}\right]$. For every such $h$, we have that

$$
\begin{aligned}
E_{M}(h) & =\left\{t \geq 0: \delta_{t}=\frac{1}{h \beta_{\nu}}\right\} \backslash J=\left(\bigcap_{\alpha<1 /\left(h \beta_{\nu}\right)} A_{\alpha}\right) \backslash\left(\bigcup_{\alpha>1 /\left(h \beta_{\nu}\right)} A_{\alpha}\right) \backslash J \\
& =\left(\bigcap_{n \geq 1} A_{1 /\left(h \beta_{\nu}\right)-1 / n}\right) \backslash\left(\bigcup_{n \neq 1} A_{1 /\left(h \beta_{\nu}\right)+1 / n}\right) \backslash J .
\end{aligned}
$$


First we give an upper bound on the Hausdorff dimension of the family of sets $\left\{A_{\delta}, \delta \geq 1\right\}$. Observe that for any $j_{0}$,

$$
A_{\delta} \subset \bigcup_{j \geq j_{0}} \bigcup_{\substack{s \in J \\ 2^{-j-1} \leq|z(s)|<2^{-j}}}\left(s-|z(s)|^{\beta_{\nu} \delta}, s+|z(s)|^{\beta_{\nu} \delta}\right) .
$$

We can use these intervals as a covering system of $A_{\delta}$. It suffices to show that for every $s>1 / \delta$, almost surely,

$$
\sum_{j \geq j_{0}}\left(2^{-j \beta_{\nu} \delta}\right)^{s} N\left([0,1] \times[0,1 / c] \times\left\{z: 2^{-j-1} \leq|z|<2^{-j}\right\}\right)<\infty,
$$

where $c$ is the constant in (3.4). This implies that for all $\delta \geq 1$ we have $\operatorname{dim}_{H} A_{\delta} \leq 1 / \delta$, almost surely.

Next we prove (3.9). Note that $N_{j}:=N\left([0,1] \times[0,1 / c] \times\left\{z: 2^{-j-1} \leq|z|<2^{-j}\right\}\right)$ is a Poisson random variable with parameter $C_{j} / c$, where $C_{j}=\int_{2^{-j-1}<|z| \leq 2^{-j}} \nu(z) \mathrm{d} z$ as in the previous section. Let $r \in\left(\beta_{\nu}, \beta_{\nu} \delta s\right)$. Then by the definition of $\beta_{\nu}$ we have $C_{j} \leq 2^{j r}$, for all $j$ large enough. Hence by the Markov inequality,

$$
\mathbb{P}\left(N_{j} \geq 2 \cdot 2^{j r}\right) \leq \mathbb{P}\left(\left|N_{j}-C_{j}\right| \geq 2^{j r}\right) \leq 2^{-j r} .
$$

It then follows by the Borel-Cantelli lemma that $N_{j} \leq 2^{j r}$ almost surely, for all $j$ sufficiently large. The convergence of the series follows, since we choose $r<\beta_{\nu} \delta s$.

The above combined with (3.8) implies that $\operatorname{dim}_{H} E_{M}(h) \leq \frac{1}{\beta_{\nu} \delta}$. To obtain a lower bound on the spectrum, we make use of the following general result; for a proof see [20]. Let $|\cdot|$ denote Lebesgue measure in $\mathbb{R}$.

Theorem 3.4. Let $\left(\lambda_{n}, \varepsilon_{n}\right)$ be a family of points, with $\lambda_{n} \in[0,1]$ and $\varepsilon_{n}>0$. Define $G_{\delta}=$ $\limsup _{n \rightarrow \infty}\left(\lambda_{n}-\varepsilon_{n}^{\delta}, \lambda_{n}+\varepsilon^{\delta}\right)$. If $\left|G_{1}\right|=1$, then for all $\delta \geq 1$

$$
\mathcal{H}^{\phi_{\delta}}\left(G_{\delta}\right)>0,
$$

where $\phi_{\delta}(x)=x^{1 / \delta}|\log x|^{2}$, and $\mathcal{H}^{\phi_{\delta}}(E)$ is the Hausdorff measure of the set $E$ with respect to the gauge function $\phi_{\delta}$.

In order to apply the above result, one would need to prove the almost-covering $\left|A_{1} \cap[0,1]\right|=1$ at critical index $\delta=1$, which holds for regular Lévy measures such as the isotropic $\alpha$-stable case with $\nu(z)=|z|^{-\alpha-d}$. However, for some ill-behaved Lévy measures the situation $\left|A_{1} \cap[0,1]\right|<1$ may occur. To overcome this, we use a trick from [42, Prop. 3.2] to construct a family of limsup sets $A_{\delta}^{*}$ embedded in $A_{\delta}$, in the sense that for every $\delta^{\prime}<\delta$,

$$
A_{\delta} \subset A_{\delta}^{*} \subset A_{\delta^{\prime}}
$$

satisfying

$$
\left|A_{1}^{*} \cap[0,1]\right|=1 .
$$

Using (3.10), we can express $E_{M}(h)$ in (3.8) with all $A_{\delta}$ replaced by $A_{\delta}^{*}$. A use of (3.11) then implies that $\mathcal{H}^{\phi_{\delta}}\left(A_{\delta}^{*}\right)>0$ almost surely, for all $\delta \geq 1$. Recalling that $\operatorname{dim}_{\mathrm{H}} A_{\delta}^{*} \leq 1 / \delta$, we have $\mathcal{H}^{\phi_{1 /\left(h \beta_{\nu}\right)}}\left(A_{1 /\left(h \beta_{\nu}\right)+1 / n}^{*}\right)=0$, which gives that $\mathcal{H}^{\phi_{1 /\left(h \beta_{\nu}\right)}}\left(E_{M}(h)\right)>0$. This proves that $D_{M}(h) \geq \beta_{\nu} h$ almost surely, simultaneously for all $0 \leq h \leq 1 / \beta_{\nu}$, as required. 
To complete the argument, it remains to construct the sets $A_{\delta}^{*}$ satisfying (3.10)-(3.11). For all integers $m<n \leq \infty$, let

$$
A_{\delta}^{m, n}=\bigcup_{\substack{s \in J \\ 2^{-n} \leq|z(s)|<2^{-m}}}\left(s-|z(s)|^{\beta_{\nu} \delta}, s+|z(s)|^{\beta_{\nu} \delta}\right) .
$$

Set $m_{1}=1$. Due to Lemma 3.2 , there exists $m_{2}>m_{1}$ such that

$$
[0,1] \subset A_{1-\frac{1}{2}} \subset A_{1-\frac{1}{2}}^{m_{1}, \infty} \text { and }\left|A_{1-\frac{1}{2}}^{m_{1}, m_{2}}\right| \geq \frac{1}{2} .
$$

Similarly, there exists $m_{3}>m_{2}$ such that

$$
[0,1] \subset A_{1-\frac{1}{3}} \subset A_{1-\frac{1}{3}}^{m_{2}, \infty} \text { and }\left|A_{1-\frac{1}{3}}^{m_{2}, m_{3}}\right| \geq 1-\frac{1}{3} .
$$

We define a sequence $\left(m_{j}\right)_{j \geq 1}$ inductively such that for all $j \geq 2,\left|A_{1-1 / j}^{m_{j-1}, m_{j}}\right| \geq 1-1 / j$. Hence,

$$
\left|\limsup _{j \rightarrow \infty} A_{1-1 / j}^{m_{j-1}, m_{j}} \cap[0,1]\right| \geq \limsup _{j \rightarrow \infty}\left|A_{1-1 / j}^{m_{j-1}, m_{j}} \cap[0,1]\right|=1 .
$$

Define $A_{\delta}^{*}=\lim \sup _{j \rightarrow \infty} A_{\delta(1-1 / j)}^{m_{j}, m_{j+1}}$; then the above formula shows (3.11). To show property (3.10), note that $A_{\delta}=\limsup _{j \rightarrow \infty} A_{\delta}^{m_{j}, m_{j+1}}$, and the first inclusion then follows. The second inclusion also holds since for every $\delta^{\prime}<\delta$ we have $\delta^{\prime}<\delta(1-1 / j)$, for all sufficiently large $j$. This completes the proof.

\subsection{Concluding remarks}

As seen from the proof, the only case when we require some extra smoothness condition for the ground state is when the Blumenthal-Getoor index is $\beta_{\nu}<1$ and $\sigma=0$. As said in Remark 3.1 this is known to hold in some cases, and it can be expected further to hold more widely.

We conjecture that the multifractal nature of a GST process will change if the ground state is less regular and $\nabla \ln \varphi_{0}$ is $C^{\varepsilon}$, with $1+\varepsilon<1 / \beta_{\nu}$. To see this, consider a simple representation for a GST process when $\beta_{\nu}<1$ and $\sigma=0$. In such cases the process has finite variation, thus the compensated Poisson integral can be decomposed in a difference of an uncompensated Poisson integral and a drift term. More precisely, the GST process is a weak solution of the simple SDE with jumps

$$
M_{t}=M_{0}+\int_{0}^{t} b\left(M_{s}\right) \mathrm{d} s+\int_{0}^{t} \int_{\mathbb{R}_{*}^{d}} z N(\mathrm{~d} s, \mathrm{~d} z),
$$

where $N$ is a Poisson measure with intensity $\mathrm{d} t \nu(z) \mathrm{d} z$, and $b(x)=\nabla \ln \varphi_{0}(x)+\left(\int_{|z| \leq 1} z \nu(z) \mathrm{d} z\right) x$ is the drift coefficient. Recall that the Hölder exponent of the pure jump Lévy term is equal to $1 /\left(\delta_{t} \beta_{\nu}\right)$.

Define the point processes

$$
\begin{aligned}
& p=\{(s, z(s)) ; s \in D\} \\
& \widetilde{p}=\{(s, r(s)) ; s \in D\} \text { with } r(s)=b\left(M_{s-}+z(s)\right)-b\left(M_{s-}\right),
\end{aligned}
$$

and the associated approximation rates $\delta_{t}$ (for $p$ ) and $\widetilde{\delta}_{t}$ (for $\widetilde{p}$ ) as in (3.6). The Hölder exponent of the drift term will depend on $\widetilde{p}$. Indeed, for the process $b\left(M_{t}\right)=G_{t}$ Lemma 3.1 implies $H_{G}(t) \leq$ $1 /\left(\beta_{\nu} \widetilde{\delta}_{t}\right)$. When $b$ is only $C^{\varepsilon}$, the jump size $r(s) \leq z(s)^{\varepsilon}$. Therefore, $H_{G}(t) \leq \varepsilon /\left(\beta_{\nu} \delta_{t}\right)$ whenever $r(s) \asymp z(s)^{\varepsilon}$ occurs for infinitely many $s$ tending to $t$.

On the other hand, in [1] it is shown that when the Hölder exponent of a Lévy process is less than $1 /\left(2 \beta_{\nu}\right)$ at some point $t$, or equivalently $\delta_{t}>2$, the time $t$ can not be an oscillating singularity 
in the sense that its primitive must have Hölder exponent $1+1 /\left(\delta_{t} \beta_{\nu}\right)$ at time $t$. It is tempting to expect that the drift term here has Hölder exponent at most $1+\varepsilon /\left(\delta_{t} \beta_{\nu}\right)$ as long as $\delta_{t}>2$, for instance, equal to 3 , and $r(s) \asymp z(s)^{\varepsilon}$ occurs for infinitely many $s$ tending to $t$. If such a $t$ exists, we get $1+\varepsilon /\left(3 \beta_{\nu}\right)<1 /\left(3 \beta_{\nu}\right)$ for $\varepsilon$ sufficiently small. This implies $H_{M}(t) \leq 1+\varepsilon /\left(3 \beta_{\nu}\right)$, and changes the singularity sets $E_{M}(h)$ for $h \leq 1+\varepsilon /\left(3 \beta_{\nu}\right)$.

Acknowledgments: JL thanks IHES, Bures-sur-Yvette, for a visiting fellowship, where part of this paper has been written. XY wishes to thank Prof. Yimin Xiao for stimulating discussions.

\section{References}

[1] P. Balança: Fine regularity of Lévy processes and linear (multi)fractional stable motion. Electron. J. Probab., 19, 1-37, 2014

[2] J. Barral, N. Fournier, S. Jaffard, S. Seuret: A pure jump Markov process with a random singularity spectrum, Ann. Probab. 38, 1924-1946, 2010

[3] J. Barral, S. Seuret: The singularity spectrum of Lévy processes in multifractal time, Adv. Math. 14, 437-468, 2007

[4] J. Bertoin: On nowhere differentiability for Lévy processes, Stoch. Stoch. Rep. 50, 205-210, 1994

[5] V. Betz, J. Lőrinczi: Uniqueness of Gibbs measures relative to Brownian motion, Ann. I.H. Poincaré 39, 877-889, 2003

[6] R.M. Blumenthal, R.K. Getoor: Sample functions of stochastic processes with stationary independent increments, J. Math. Mech. 10, 493-516, 1961

[7] B. Böttcher: On the construction of Feller processes with unbounded coefficients, Elect. Comm. Probab. 16, $545-555,2011$

[8] B. Böttcher, R. Schilling, J. Wang: Lévy-Type Processes: Construction, Approximation and Sample Path Properties, Lecture Notes in Mathematics 2099, Lévy Matters vol. III, 2013

[9] Ph. Courrège: Générateur infinitésimal d'un semi-groupe de convolution sur $\mathbb{R}^{n}$ et formule de Lévy-Khintchine, Bull. Sc. Math., 2e série, 88, 3-30, 1964

[10] A. Durand, S. Jaffard: Multifractal analysis of Lévy fields, Probab. Theory Relat. Fields 153, 45-96, 2012

[11] S.O. Durugo, J. Lörinczi: Spectral properties of the quartic massless relativistic oscillator, J. Diff. Equations 264, 3775-3809, 2018

[12] F. Hiroshima, T. Ichinose, J. Lörinczi: Path integral representation for Schrödinger operators with Bernstein functions of the Laplacian, Rev. Math. Phys. 24, 1250013, 2012

[13] F. Hiroshima, T. Ichinose, J. Lörinczi: Probabilistic representation and fall-off of bound states of relativistic Schrödinger operators with spin 1/2, Publ. Res. Inst. Math. Sci., 49, 2013, 189-214

[14] W. Hoh: Pseudo-Differential Operators Generating Markov Processes, Habilitationsschrift, Universität Bielefeld, 1998

[15] N. Ikeda, S. Watanabe: Stochastic Differential Equations and Diffusion Processes, North Holland, 1981

[16] N. Jacob: Pseudo-Differential Operators and Markov Processes, vols. 1-3, Imperial College Press, 2003-2005

[17] J. Jacod, A.N. Shiryaev: Limit Theorems for Stochastic Processes, Springer, 2nd ed., 2003

[18] S. Jaffard: Old friends revisited: the multifractal nature of some classical functions, J. Fourier Anal. Appl. 3, $1-22,1997$

[19] S. Jaffard: The multifractal nature of Lévy processes, Probab. Theory Rel. Fields 114, 207-227, 1999

[20] S. Jaffard: On lacunary wavelet series, Ann. Appl. Probab. 10, 313-329, 2000

[21] K. Kaleta, J. Lörinczi: Fractional $P(\phi)_{1}$-processes and Gibbs measures, Stoch. Proc. Appl. 122, 3580-3617, 2012

[22] K. Kaleta, J. Lőrinczi: Pointwise eigenfunction estimates and intrinsic ultracontractivity-type properties of Feynman-Kac semigroups for a class of Lévy processes, Ann. Probab. 43, 1350-1398, 2015

[23] K. Kaleta, J. Lörinczi: Transition in the decay rates of stationary distributions of Lévy motion in an energy landscape, Phys. Rev. E 93, 022135, 2016

[24] K. Kaleta, J. Lőrinczi: Fall-off of eigenfunctions for non-local Schrödinger operators with decaying potentials, Potential Anal. 46, 647-688, 2017

[25] K. Kaleta, J. Lörinczi: Typical long time behaviour of ground state-transformed jump processes, to appear in Commun. Contemp. Math.

[26] D. Khoshnevisan, R.L. Schilling, Y. Xiao: Packing dimension profiles and Lévy processes, Bull. London Math. Soc. 44, 931-943, 2012

[27] D. Khoshnevisan, Y. Xiao: Lévy processes: capacity and Hausdorff dimension, Ann. Probab. 33, 841-878, 2005 
[28] D. Khoshnevisan, Y. Xiao: Packing dimension of the range of a Lévy process, Proc. Amer. Math. Soc. 136, 2597-2607, 2008

[29] V.N. Kolokoltsov: Nonlinear Markov Processes and Kinetic Equations, Cambridge University Press, 2010

[30] T. Kurtz: Equivalence of stochastic equations and martingale problems, in: Stochastic Analysis 2010, D. Crisan (ed.), pp 113-130, 2010

[31] F. Kühn: Solutions of Lévy-driven SDEs with unbounded coefficients as Feller processes, Proc. Amer. Math. Soc. 146, 3591-3604, 2018

[32] J. Lörinczi, F. Hiroshima, V. Betz: Feynman-Kac-Type Theorems and Gibbs Measures on Path Space. With Applications to Rigorous Quantum Field Theory, de Gruyter Studies in Mathematics 34, Walter de Gruyter, 2011; 2nd extended ed. to appear in 2019

[33] J. Lörinczi, J. Małecki: Spectral properties of the massless relativistic harmonic oscillator, J. Diff. Equations 253, 2846-2871, 2012

[34] M.M. Meerschaert, Y. Xiao: Dimension results for sample paths of operator stable Lévy processes, Stoch. Proc. Appl. 115, 55-75, 2005

[35] S. Orey, S.J. Taylor: How often on a Brownian path does the law of iterated logarithm fail?, Proc. London Math. Soc. 28, 174-192, 1974

[36] R.L. Schilling: Growth and Hölder conditions for the sample paths of Feller processes, Probab. Theory Rel. Fields 112, 565-611, 1998

[37] R.L. Schilling, A. Schnurr: The symbol associated with the solution of a stochastic differential equation, Elect. J. Probab. 15, 1369-1393, 2010

[38] M.A. Schwarzenberger: Affine Processes and Pseudo-Differential Operators with Unbounded Coefficients, PhD thesis, TU Dresden, 2016

[39] L.A. Shepp: Covering the line with random intervals, Z. Wahrscheinlichkeitstheorie verw. Geb. 23, 163-170, 1972

[40] B. Simon: Functional Integration and Quantum Physics, Academic Press, 1976, AMS Chelsea Publishing, 2004, 2nd ed.

[41] Y. Xiao: Random fractals and Markov processes, in: Fractal Geometry and Applications: a Jubilee of Benoit Mandelbrot, Proc. Sympos. Pure Math. AMS, vol. 72, Providence, RI, 2004, pp 261-338

[42] L. Xu: The multifractal nature of Boltzmann processes, Stoch. Proc. Appl. 126, 2181-2210, 2016

[43] X. Yang: Hausdorff dimension of the range and the graph of stable-like processes, J. Theor. Probab., to appear

[44] X. Yang: Étude dimensionnelle de la régularité de processus de diffusion à sauts (Dimension Properties of the Regularity of Jump Diffusion Processes), PhD thesis, Université Paris-Est, 2016

József Lörinczi, Department of Mathematical Sciences, Loughborough University, Loughborough LE11 3TU, UNITED KINGDOM

E-mail address: J.Lorinczi@lboro.ac.uk

Xiaochuan Yang, Department of Statistics and Probability, Michigan State University, 619 Red Cedar Road, East Lansing, MI, USA

E-mail address: yangxi43@stt.msu.edu, xiaochuan.j.yang@gmail.com 